\title{
Evolution of Balmer jump selected galaxies in the ALHAMBRA survey ${ }^{\star}$
}

\author{
P. Troncoso Iribarren ${ }^{1,2}$, L. Infante ${ }^{1,2}$, N. Padilla ${ }^{1,2}$, I. Lacerna ${ }^{1,2,5}$, S. Garcia ${ }^{1,2}$, A. Orsi ${ }^{3}$, A. Muñoz Arancibia ${ }^{1,14}$, \\ J. Moustakas ${ }^{4}$, D. Cristóbal-Hornillos ${ }^{3}$, M. Moles $^{3,6}$, A. Fernández-Soto ${ }^{9,10}$, V. J. Martínez ${ }^{9,11,12}$, M. Cerviño ${ }^{6,7,8}$, \\ E. J. Alfaro ${ }^{6}$, B. Ascaso ${ }^{13,15}$, P. Arnalte-Mur ${ }^{11,12}$, L. Nieves-Seoane ${ }^{10,11}$, and N. Benítez ${ }^{6}$ \\ 1 Instituto de Astrofísica, Pontificia Universidad Católica de Chile, Avda. Vicuña Mackenna 4860, 782-0436 Macul, Santiago, Chile \\ e-mail: ptroncos@astro.puc.cl \\ 2 Centro de Astro-Ingeniería, Pontificia Universidad Católica de Chile, Avda. Vicuña Mackenna 4860, 782-0436 Macul, Santiago, \\ Chile \\ 3 Centro de Estudios de Física del Cosmos de Aragón, Plaza de San Juan 1, 44001 Teruel, Spain \\ 4 Department of Physics and Astronomy, Siena College, 515 Loudon Road, Loudonville, NY 12211, USA \\ 5 Max Planck Institute for Astronomy, Königstuhl 17, 69117 Heidelberg, Germany \\ 6 Instituto de Astrofísica de Andalucía (IAA-CSIC), Glorieta de la Astronomía, 18008 Granada, Spain \\ 7 Instituto de Astrofísica de Canarias, Vía Láctea s/n, 38200 La Laguna, Tenerife, Spain \\ 8 Departamento de Astrofísica, Facultad de Física, Universidad de La Laguna, 38206 La Laguna, Spain \\ 9 Unidad Asociada Observatorio Astronómico (IFCA-UV), 46980 Paterna, Spain \\ 10 Instituto de Física de Cantabria (CSIC-UC), 39005 Santander, Spain \\ 11 Observatori Astronòmic, Universitat de València, C/ Catedràtic José Beltrán 2, 46980 Paterna, Spain \\ 12 Departament d'Astronomia i Astrofísica, Universitat de València, 46100 Burjassot, Spain \\ 13 GEPI, Observatoire de Paris, CNRS, Université Paris Diderot, 61, Avenue de l'Observatoire 75014 Paris, France \\ 14 Instituto de Física y Astronomía, Universidad de Valparaíso, Avda. Gran Bretaña 1111, Valparaíso, Chile \\ 15 APC, AstroParticule et Cosmologie, Université Paris Diderot, CNRS/IN2P3, CEA/lrfu, Observatoire de Paris, \\ Sorbonne Paris Cité, 10 rue Alice Domon et Léonie Duquet, 75205 Paris Cedex 13, France
}

Received 13 October 2015 / Accepted 30 January 2016

\section{ABSTRACT}

Context. Samples of star-forming galaxies at different redshifts have been traditionally selected via color techniques. The ALHAMBRA survey was designed to perform a uniform cosmic tomography of the Universe, and we here exploit it to trace the evolution of these galaxies.

Aims. Our objective is to use the homogeneous optical coverage of the ALHAMBRA filter system to select samples of star-forming galaxies at different epochs of the Universe and study their properties.

Methods. We present a new color-selection technique, based on the models of spectral evolution convolved with the ALHAMBRA bands and the redshifted position of the Balmer jump to select star-forming galaxies in the redshift range $0.5<z<1.5$. These galaxies are dubbed Balmer-jump Galaxies (BJGs). We applied the iSEDfit Bayesian approach to fit each detailed spectral energy distribution and determined star-formation rate (SFR), stellar mass, age, and absolute magnitudes. The mass of the halos in which these samples reside were found through a clustering analysis.

Results. Five volume-limited $B J G$ subsamples with different mean redshifts are found to reside in halos of median masses $\sim 10^{12.5 \pm 0.2} M_{\odot}$ slightly increasing toward $z=0.5$. This increment is similar to numerical simulations results, which suggests that we trace the evolution of an evolving population of halos as they grow to reach a mass of $\sim 10^{12.7 \pm 0.1}$ at $z=0.5$. The likely progenitors of our samples at $z \sim 3$ are Lyman-break galaxies, which at $z \sim 2$ would evolve into star-forming $B z K$ galaxies, and their descendants in the local Universe are galaxies with luminosities of $1-3 L_{*}$. Hence, this allows us to follow the putative evolution of the SFR, stellar mass, and age of these galaxies.

Conclusions. From $z \sim 1.0$ to $z \sim 0.5$, the stellar mass of the volume-limited BJG samples changes almost not at all with redshift, suggesting that major mergers play a minor role in the evolution of these galaxies. The SFR evolution accounts for the small variations of stellar mass, suggesting that star formation and possible minor mergers are the main channels of mass assembly.

Key words. Galaxy: evolution - Galaxy: halo - galaxies: high-redshift - galaxies: evolution - galaxies: general - galaxies: photometry

\section{Introduction}

Most of the recent observational efforts to understand galaxy evolution have been focused on determining the history of cosmic star formation, gas density evolution, metallicity evolution, and mass growth of the Universe (Daddi et al. 2004; Mannucci et al. 2010; Madau \& Dickinson 2014; Tomczak et al. 2014; Bouwens et al. 2015). These multiwavelength

\footnotetext{
* Based on data obtained at the Calar Alto Observatory.
}

observational constraints have usually been summarized as galaxy scaling relations that might or might not change with redshift (Mannucci et al. 2010; Elbaz et al. 2011; Bouwens et al. 2014; Troncoso et al. 2014), in high- or low-density environments, in extreme physical conditions (starburst, AGN galaxies), and in spatially resolved data due to internal variations of the galaxy properties (Sanchez et al. 2013). In parallel, theoretical works and simulations have tried to explain the physical mechanisms that reproduce the measured global properties 
(Daddi et al. 2010; Davé et al. 2011; Lilly et al. 2013; Lagos et al. 2014; Padilla et al. 2014). Despite these efforts, the completeness and cleanness of the sample are still challenging problems that depend on the sample selection-method, instrument limits, and telescope time. These problems make the comparison between observational and theoretical works even more difficult. For example, Campbell et al. (2014) compared the stellar mass of GALFORM galaxies predicted by the model with those obtained through the fit of their predicted broad-band colors. They found that both quantities differ for an individual galaxy, hence the clustering of mass-selected samples can be affected by systematic biases. Therefore, mass-selected samples might provide erroneous conclusions regarding their progenitors and descendants. In addition, the evolution of scaling relations is constrained with observations of galaxy samples that are selected with luminosity or stellar-mass thresholds and are located at different redshifts, which does not necessarily constitute causally connected populations (i.e., they do not follow a progenitor-todescendant relation). Clustering-selected samples overcome this problem because in a hierarchical clustering scenario, a correlation analysis allows us to estimate the bias and hence statistically determine the progenitors and descendants of galaxy samples. The bias parameter measures the clustering difference between the galaxy spatial distribution and underlying dark-matter distribution. Thus, it relates the typical mass of halos hosting the galaxies (Sheth et al. 2001). Hence, measuring it in galaxy samples at different redshifts determines whether we are following the evolution of baryonic processes occurring in halos of similar masses or not. This fact is of extreme importance because once it is determined, we can use the multiwavelength data to study the evolution of the baryonic processes at certain halo mass, establishing a direct link between observations and galaxy formation models. Padilla et al. (2011) selected early-type galaxies according to their clustering and luminosity function in the MUSYC survey. So far, no study that selects star-forming galaxies according their clustering and luminosity function has been reported.

Star-forming galaxies are of particular interest because they allow us to study the mechanisms that switch the star formation on or off and its evolution with redshift. Considering the lack of wide spectroscopic surveys in the sense of wavelength coverage and surveyed area, the majority of the star-forming galaxy samples have been chosen using two-color selection techniques. The so-called drop-out technique is based on recording the difference between two distinct parts of the spectrum that generate a break on it (e.g., the $912 \AA$ Lyman break and the $3646 \AA$ Balmer jump). This difference is strong enough that it has been measured in broad bands, selecting star-forming galaxies at early periods of the Universe $(z>1.4)$, such as the BzK, BX, BM, DOGs, and LBGs (Steidel et al. 1996; Daddi et al. 2004; Steidel et al. 2004; Dey et al. 2008; Infante et al. 2015). Several authors have measured the bias (Gawiser et al. 2007; Blanc et al. 2008; Guaita et al. 2010) by determining the mass of the halo in which each galaxy sample resides and by connecting the progenitors and descendants of these galaxy samples. Other works selected the samples by fully relying on their photometric redshift and the physical properties determined through fitting the spectral energy distribution (SED; Tomczak et al. 2014), far-IR luminosity (Rodighiero et al. 2011), or Bayesian approaches such as iSEDfit (Moustakas et al. 2013). Recently, Viironen et al. (2015) implemented a method in the ALHAMBRA survey to select galaxy samples using the probability distribution of the photometric redshift ( $z \mathrm{PDF}$ ). The quality of the detailed SED distribution, provided by the medium bands of the ALHAMBRA survey, allowed them to perform an accurate statistical analysis.
They included our lack of knowledge of the precise galaxy redshift and selected the sample according to a certain probability threshold defined by the authors. Therefore, this method by definition selects a clean but not a complete sample.

In this work, we aim to develop a technique based purely on photometric data to select star-forming galaxies. This type of selection allows us to directly compare our results with the previously mentioned works that also used a drop-out technique to select their BzK, LBG, etc. samples. We use the uniform separation between two contiguous medium bands of the ALHAMBRA survey to register the redshifted position of the $3646 \AA$ Balmer jump within the optical domain, allowing us to select galaxy samples in the redshift range $0.5<z<2$.

We based our two-color selection technique on the Bruzual \& Charlot (2003) models and applied it to the GOLD ALHAMBRA catalogs (Molino et al. 2014). In the following, the galaxies selected by this method are dubbed Balmer-jump Galaxies (BJGs), and their physical properties are investigated. Based on a clustering study, we find the progenitors and descendants of galaxies in these samples, allowing us to study the evolution of the properties, derived through a SED fit, as a function of redshift for halos of a certain mass. This paper is organized as follows: in Sect. 2 we summarize the ALHAMBRA observations and introduce the nomenclature of the ALHAMBRA filter system used throughout the paper. In Sect. 3 we describe the selection method and justify it on the Bruzual \& Charlot (2003) models. The BJG samples are defined here. In Sect. 4 each galaxy SED is modeled using iSEDfit (Moustakas et al. 2013), and the physical properties of each sample are characterized as a whole. In Sect. 5 the clustering properties are calculated, and in Sect. 6 we discuss the main results. Finally, we conclude in Sect. 7. Throughout the paper, we use a standard flat cosmology with $H_{0}=100 h \mathrm{~km} \mathrm{~s}^{-1} \mathrm{Mpc}^{-1}, \Omega_{\mathrm{m}}(z=0)=0.3$, $\Omega_{\Lambda}(z=0)=0.7, \sigma_{8}=0.824 \pm 0.029$, and the magnitudes are expressed in the $\mathrm{AB}$ system.

\section{Data: the ALHAMBRA survey}

The Advanced Large Homogeneous Area Medium-Band Redshift Astronomical (ALHAMBRA ${ }^{1}$ ) survey provides a kind of cosmic tomography for the evolution of the contents of the Universe over most of the cosmic history. Benitez et al. (2009) have especially designed a new optical photometric system for the ALHAMBRA survey that maximizes the number of objects with an accurate classification by SED and photometric redshift. It employs 20 contiguous, equal-width $\sim 310 \AA$, medium bands that cover the wavelength range from $3500 \AA$ to $9700 \AA$, plus the standard $J H K$ near-infrared bands. Moles et al. (2008) and Aparicio Villegas et al. (2010) presented an extensive description of the survey and filter transmission curves. The observations were made at Calar Alto Observatory (CAHA, Spain) with the $3.5 \mathrm{~m}$ telescope using the two wide-field imagers in the optical (LAICA) and NIR (Omega-2000). The total surveyed area is $2.8 \mathrm{deg}^{2}$ distributed in eight fields that overlap areas of other surveys such as SDSS, COSMOS, DEEP-2, and HDF-N. The typical seeing of the optical images is $1.1 \mathrm{arcsec}$, while for the NIR images it is 1.0 arcsec. For details about the survey and data release we refer to Molino et al. (2014) and Cristóbal-Hornillos et al. (2009). We here used the public GOLD catalogs ${ }^{2}$, which contains data of seven out of the eight ALHAMBRA fields. The magnitude limits of these catalogs are $\left\langle m_{\mathrm{AB}}\right\rangle \sim 25$ for the four

\footnotetext{
http://www.alhambrasurvey.com

http://cosmo.iaa.es/content/ALHAMBRA-Gold-catalog
} 
Table 1. Name and effective wavelength of each ALHAMBRA filter.

\begin{tabular}{lccccc}
\hline \hline Name & $\begin{array}{c}\lambda_{\text {eff }} \\
{[\mathrm{nm}]}\end{array}$ & Name & $\begin{array}{c}\lambda_{\text {eff }} \\
{[\mathrm{nm}]}\end{array}$ & Name & $\begin{array}{c}\lambda_{\text {eff }} \\
{[\mathrm{nm}]}\end{array}$ \\
\hline$U_{1}$ & 365.5 & $U_{2}$ & 396.5 & $B_{3}$ & 427.5 \\
$B_{4}$ & 458.5 & $B_{5}$ & 489.5 & $B_{6}$ & 520.5 \\
$B_{7}$ & 551.5 & $R_{8}$ & 582.5 & $R_{9}$ & 613.5 \\
$R_{10}$ & 644.5 & $R_{11}$ & 675.5 & $R_{12}$ & 706.5 \\
$I_{13}$ & 737.5 & $I_{14}$ & 768.5 & $I_{15}$ & 799.5 \\
$I_{16}$ & 830.5 & $z_{17}$ & 861.5 & $z_{18}$ & 892.5 \\
$z_{19}$ & 923.5 & $z_{20}$ & 954.5 & & \\
\hline
\end{tabular}

Notes. Columns 1, 3, and 5 indicate the adopted filter name that is used throughout the paper; Cols. 2, 4, and 6 show the effective wavelength of each ALHAMBRA band.

blue bands and from $\left\langle m_{\mathrm{AB}}\right\rangle \sim 24.7 \mathrm{mag}$ to $23.4 \mathrm{mag}$ for the red bands. The NIR limits at $\mathrm{AB}$ magnitudes are $J \approx 24 \mathrm{mag}$, $H \approx 23 \mathrm{mag}$, and $K \approx 22 \mathrm{mag}$. In the following, the filter nomenclature presented in Table 1 is used and the ALH-4 and ALH-7 fields are excluded from our analysis. Previous authors have shown that overdensities reside in these fields, which might alter the redshift distribution of the selected samples as well as the clustering measurements (see Sect. 5). Arnalte-Mur et al. (2014) obtained the clustering properties of ALHAMBRA galaxies and studied the sample variance using the seven independent ALHAMBRA fields. They quantified the impact of individual fields on the final clustering measurements. Using the Norberg et al. (2011) method, they determined two outliers regions, which are the ALH-4 and ALH-7 fields. In addition, part of the ALH-4 field spatially corresponds to the COSMOS field. Previous works have shown that this region is dominated by some large-scale structures (LSS), the most prominent are peaking at $z \sim 0.7$, and 0.9 (Scoville et al. 2007). These structures have X-ray counterparts and a probability higher than $30 \%$ of being LSS. Guzzo et al. (2007) studied the clusters located at the center of the LSS (at $z \sim 0.7$ ), while Finoguenov et al. (2007) found diffuse X-ray emission in the most compact structures. There are other LSS found in the COSMOS field, but they fall out of the redshift range of the samples studied in this paper.

\section{Sample selection}

To exclude the stars from the original ALHAMBRA Gold catalog, we used the stellarity index given by SExtractor (CLASS-STAR parameter $C(K)$ ) and the statistical star and galaxy separation (Molino et al. 2014, Sect. 3.6) encoded in the variable stellar flag $\left(S_{\text {alh }}\right)$ of the catalogs. Throughout the present paper, we define as galaxies those ALHAMBRA sources with $C(K) \leq 0.8$ and $S_{\text {alh }} \leq 0.5$. The ALHAMBRA Gold catalog is an $F 814 W$ (i.e., almost an I-band) selected catalog. This band was created by the ALHAMBRA team as a linear combination of ALHAMBRA bands (see Eq. (5) in Molino et al. 2014), and it was used for their source detection. Objects with faint features in this band are not detected, which might affect the completeness of the selected sample. We discuss this in more detail in Sects. 4.3 and 6.1. This catalog is complete up to $F 814 W=23$ (Molino et al. 2014), hence we used this limit to build our complete sample. Cristóbal-Hornillos et al. (2009) studied the NIR completeness using the early release of the first ALHAMBRA field. They determined a change in the slope of the $K$-band number counts in the magnitude range $18.0<K_{\text {Vega }}<20.0$

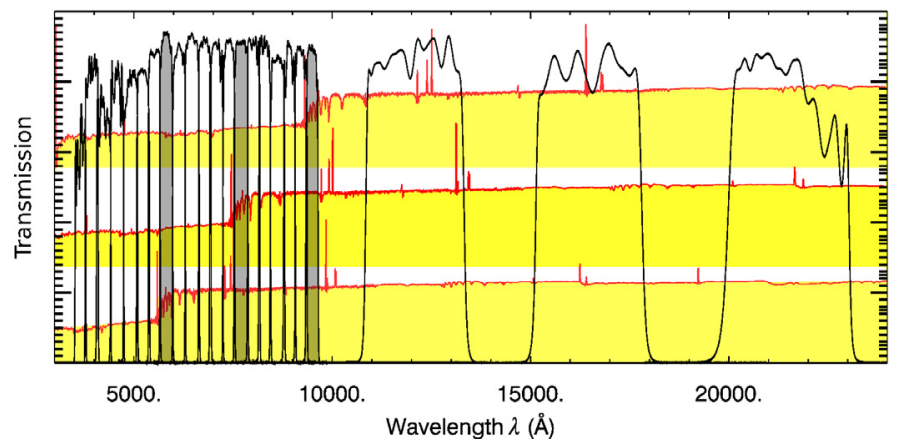

Fig. 1. ALHAMBRA filter transmission curve covering the optical to NIR. From top to bottom, the red curves show the typical redshifted spectrum of a star-forming galaxy at $z \sim 1.5, z \sim 1.0$ and $z \sim 0.5$. The gray shaded areas mark the position of the ALHAMBRA filters $R_{8}$, $I_{14}$, and $z_{20}$, which highlights the position of the Balmer jump. To select the $B J G$ samples, we use the filter directly redward of the gray filter to sample the end position of the Balmer jump.

and showed that the data are complete until $K_{\mathrm{VEGA}}=20$ or $K_{\mathrm{AB}}=21.8$. We have checked the $K$-band numbers counts of the 48 individual pointing that comprise the ALHAMBRA GOLD catalog. Every single pointing tends to fall at $K_{\mathrm{AB}} \sim 22$. We used the $K_{\mathrm{AB}}=22$ limit to define our complete samples.

\subsection{Selection of star-forming galaxies}

We used the models of Bruzual \& Charlot (2003) convolved with the ALHAMBRA filter system to define a two-color criterion to select star-forming galaxies analogously to the work of Daddi et al. (2004). They created the $B z K$ color-selection technique to cull star-forming galaxies at $z>1.4$. This technique is based on the Balmer jump, which is an indicator of recent star formation. They used the models of Bruzual \& Charlot (2003) to identify the redshifted Balmer jump in the z-band, creating the color-selection criterion $B z K \equiv(z-K)_{\mathrm{AB}}-(B-z)_{\mathrm{AB}}=-0.2$; where $B z K \geq-0.2$ selects star-forming galaxies at $z>1.4$. Our approach is analogous to the $B z K$ method in the sense that we also used the Bruzual \& Charlot (2003) models, but we recorded redshifted Balmer jumps in various (diverse) medium bands to select galaxies at different redshifts. This situation is illustrated in Fig. 1, which shows the typical spectrum of a starforming galaxy redshifted to $z \sim 0.5, z \sim 1.0$ and $z \sim 1.5$. The ALHAMBRA bands are overplotted from the optical to NIR ranges. We chose the $U_{2}$-band to sample the region before the Balmer jump instead of the $B$-band. We selected the $U_{2}$ band because it reaches a higher completeness level than $U_{1}$. Objects that were not detected in the $U_{2}$ band were also considered in our selection, and their magnitude limit was used. Redder bands might sample weak features blueward of the Balmer jump of low-redshift galaxies $z<0.4$. The $K$ band is exactly the same as was used by Daddi et al. (2004), while the z-band was replaced with the variable $X_{n}$-band, which covers the optical range from $613 \AA$ to $954 \AA$. This means that $X_{n}$ can be any ALHAMBRA filter from $R_{9}$ to $z_{20}$ (see Table 1 ). The $X_{n}$-band samples the region directly redward of the Balmer jump, hence the $U_{2}-X_{n}$ color indicates the galaxy redshift range depending on the selected $n$. On the other hand, the $X_{n}-K$ color registers the duration of the star formation age. The different panels in Figs. 2 and 3 show the theoretical evolution (Bruzual \& Charlot 2003) of the $U_{2} X_{n} K \equiv\left(X_{n}-K\right)-\left(U_{2}-X_{n}\right)$ color as a function of redshift with $9<n<20\left(X_{n}=R_{9}, \ldots ., z_{20}\right)$. Red, green, 

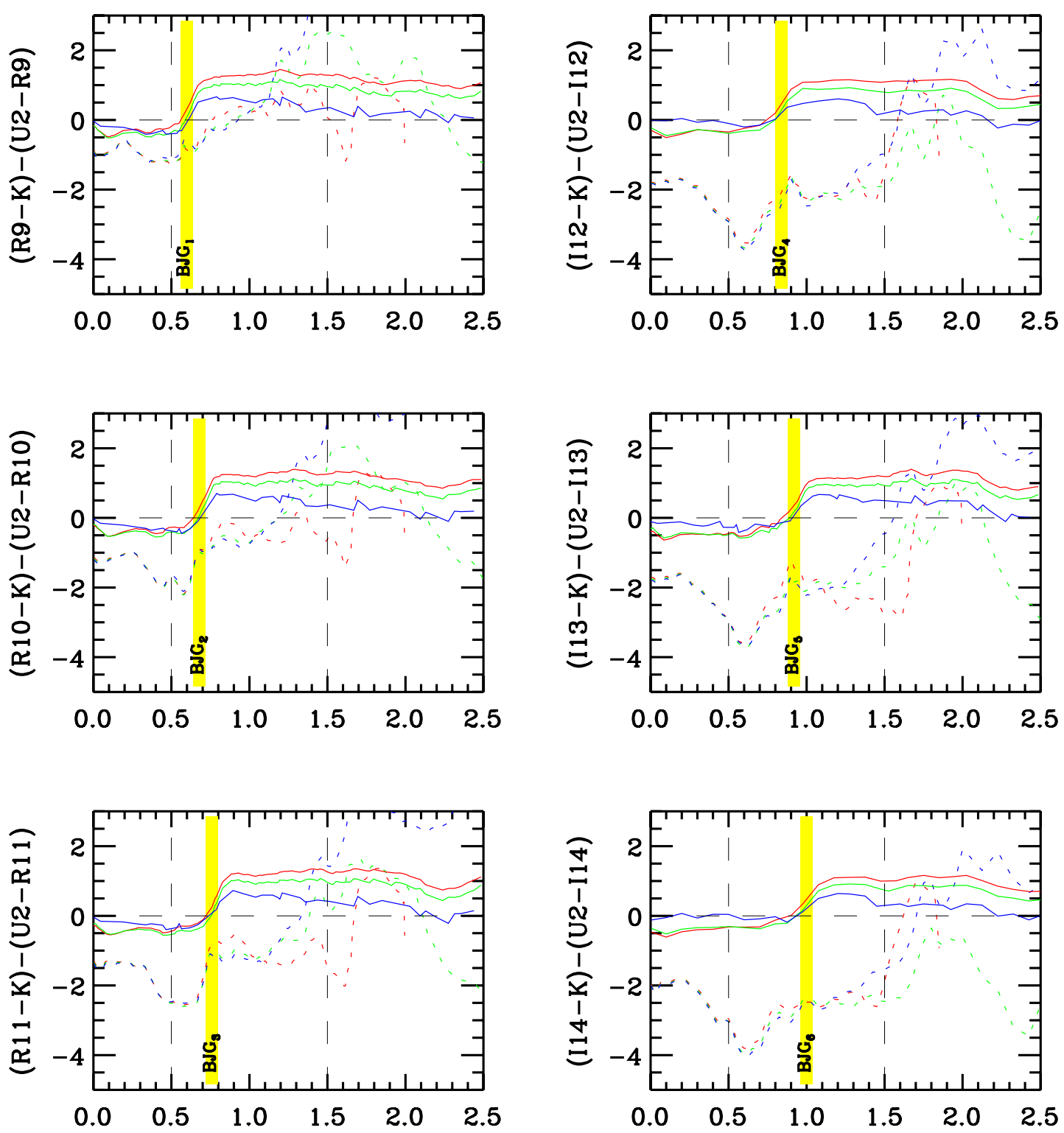

\section{Redshift}

Fig. 2. Criteria for selecting star-forming galaxies. The solid and dashed lines show the color evolution of star-forming and passive galaxies, respectively. Red, green, and blue dashed lines show the passively evolving models with a formation redshift of $z_{\mathrm{f}}=2,3,6$, respectively. Red, green, and blue solid lines show models that evolve with a constant star-formation rate for ages of $0.2,1$, and 2 Gyr. The horizontal dashed line indicates the color cut $U_{2} X_{n} K \equiv\left(U_{2}-X_{n}\right)-\left(X_{n}-K\right)=0$. In each panel, the star-forming galaxies are located above this threshold. The yellow shaded regions mark the redshift range of the selected $B J G$ samples in each color combination. Dashed vertical lines indicate the redshift 0.5 and 1.5 .

and blue solid lines show the $U_{2} X_{n} K$-color evolution of constant star-formation rate models for ages $0.2,1$, and 2 Gyr and reddening $E(B-V)=0.3$. Red, green, and blue dashed lines show the $U_{2} X_{n} K$-color evolution of passively evolving models for formation redshift of $z_{f}=2,3$ and 6 . In each panel, the star-forming models always lie in the region $U_{2} X_{n} K>0$. Consequently, to select a sample at redshift higher than $z \sim 0.5$, a combination that involves a $X_{n}$ filter redder than $R_{9}$ must be used, as we show in the bottom panels of Figs. 2 and 3.

\subsection{BJG samples}

We used the condition $U_{2} X_{n} K \equiv\left(U_{2}-X_{n}\right)-\left(X_{n}-K\right)>0$ and the homogeneous coverage in the optical range of the ALHAMBRA filter system, where $n$ ranges from 9 to $20\left(X_{n}=R_{9}, . ., z_{20}\right)$, to select galaxies at redshift $z>0.5,0.6,0.7,0.8,0.85,0.95,1.05$, $1.1,1.2,1.25$, and 1.3. In each ALHAMBRA filter set $U_{2} X_{n} K$, this color condition selects star-forming and passive galaxies in the wide redshift range. Theoretically, these selected passive galaxies always lie at higher redshift (i.e., $\Delta z>0.25$ ) than the selected star-forming galaxies. To select galaxies in a narrow redshift range, we used more than one color condition; first the $\left(U_{2}-X_{n}\right)-\left(X_{n}-K\right)>0$ condition with $n=j$, to select all galaxies above certain redshift $z_{j}$, and second we subtracted the higher redshift samples, selected with $n \geq j+1$ (passive and star forming), from the first galaxy selection. For example, our lowest redshift sample was selected by imposing the condition $U_{2} R_{9} K=0$ (i.e., select galaxies at $z>0.5$ ) and subtracting the galaxy samples selected by $U_{2} X_{n} K>0$ with $n \geq 10$, which selects all galaxies at $z>0.6$. In this way, only the star-forming galaxies 
P. Troncoso Iribarren et al.: The evolution of Balmer jump selected galaxies in the ALHAMBRA survey
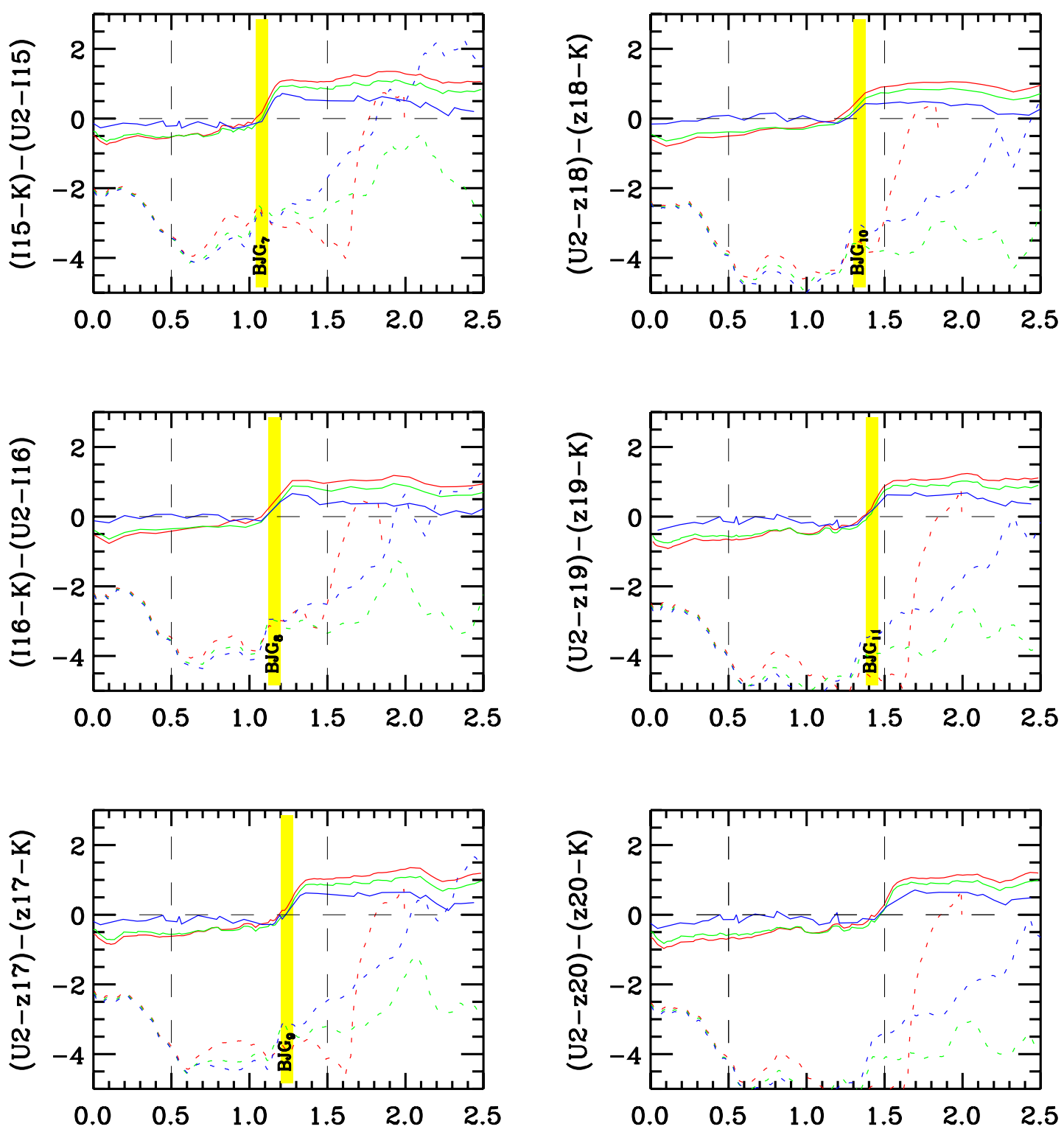

\section{Redshift}

Fig. 3. Criteria for selecting star-forming galaxies. The solid and dashed lines show the color evolution of star-forming and passive galaxies, respectively. Red, green, and blue dashed lines show the passively evolving models with a formation redshift of $z_{\mathrm{f}}=2,3,6$, respectively. Red, green, and blue solid lines show models that evolve with a constant star-formation rate for ages of $0.2,1$, and 2 Gyr. The horizontal dashed line indicates the color cut $U_{2} X_{n} K \equiv\left(U_{2}-X_{n}\right)-\left(X_{n}-K\right)=0$. In each panel, the star-forming galaxies are located above this threshold. The yellow shaded regions mark the redshift range of the selected $B J G$ samples in each color combination. Dashed vertical lines indicate the redshift 0.5 and 1.5.

in the redshift range $0.5<z<0.6$ were selected (see yellow shaded region in Figs. 2 and 3). Following this method and using the homogeneous separation between each ALHAMBRA band, we selected eleven star-forming galaxy samples peaking at $z \sim 0.55,0.65,0.75,0.8,0.9,1.0,1.05,1.15,1,2,1.25$, and $z \sim 1.4$ by culling the galaxies that satisfied the star-forming criterion $U_{2} X_{n} K \equiv\left(U_{2}-X_{n}\right)-\left(X_{n}-K\right)>0$, where $X_{n}=R_{9}, \ldots, z_{19}$ and cleaned of higher redshift galaxies by subtracting the samples that satisfied $U_{2} X_{n} K>0$, with $n \geq 10, \ldots, 20$. Table 2 summarizes the properties of the selected samples using the method described above. We ensured that all samples are roughly independent of each other by removing the high-redshift samples that were selected using the $X_{n}$ filters with $n \geq j+1$ (see Col. 3 of Table 2) from the sample selected with the filter $X_{n=j}$.

\section{Physical properties of the BJG samples}

In this section, we characterize each $B J G$ sample by providing a mean characteristic value of its redshift, absolute magnitude, stellar mass, age, star formation rate, etc.

\subsection{Photometric redshifts}

We used the Bayesian photometric redshift ( $z \mathrm{BPZ}$ ) published in the Gold ALHAMBRA catalogs (Molino et al. 2014) to verify our selection method. The BPZ code was optimized to determine photometric redshifts, for details on the $z \mathrm{BPZ}$ calculations see Molino et al. (2014) and Benitez et al. (2009). Figure 4 shows the $z \mathrm{BPZ}$ distribution of the eleven $B J G$ samples. To 
Table 2. Properties of the $B J G$ selected samples.

\begin{tabular}{lcccc}
\hline \hline $\begin{array}{l}\text { Sample } \\
\text { name }\end{array}$ & $\begin{array}{c}\text { Initial set } \\
U_{2} X_{n} K\end{array}$ & $\begin{array}{c}\text { Clean sets } \\
U_{2} X_{n} K\end{array}$ & $N$ & $\begin{array}{c}\langle z\rangle \\
\text { Two-color }\end{array}$ \\
\hline$B J G_{1}$ & $R_{9}$ & $n \geq 10$ & 5489 & $0.5-0.6$ \\
$B J G_{2}$ & $R_{10}$ & $n \geq 11$ & 5174 & $0.6-0.7$ \\
$B J G_{3}$ & $R_{11}$ & $n \geq 12$ & 4497 & $0.7-0.8$ \\
$B J G_{4}$ & $I_{12}$ & $n \geq 13$ & 4012 & $0.8-0.85$ \\
$B J G_{5}$ & $I_{13}$ & $n \geq 14$ & 3550 & $0.85-0.95$ \\
$B J G_{6}$ & $I_{14}$ & $n \geq 15$ & 2878 & $0.95-1.05$ \\
$B J G_{7}$ & $I_{15}$ & $n \geq 16$ & 2231 & $1.05-1.1$ \\
$B J G_{8}$ & $I_{16}$ & $n \geq 17$ & 2325 & $1.1-1.2$ \\
$B J G_{9}$ & $z_{17}$ & $n \geq 18$ & 2058 & $1.2-1.25$ \\
$B J G_{10}$ & $z_{18}$ & $n \geq 19$ & 2140 & $1.25-1.3$ \\
$B J G_{11}$ & $z_{19}$ & $n=20$ & 3391 & $1.3-1.5$ \\
\hline
\end{tabular}

Notes. Column 1, name of the selected sample; Col. 2, initial filter set used for selection; Col. 3, second filter sets used to subtract higher redshift galaxies from the initial sample; Col. 4, number of galaxies selected; Col. 5, expected redshift range of the sample.

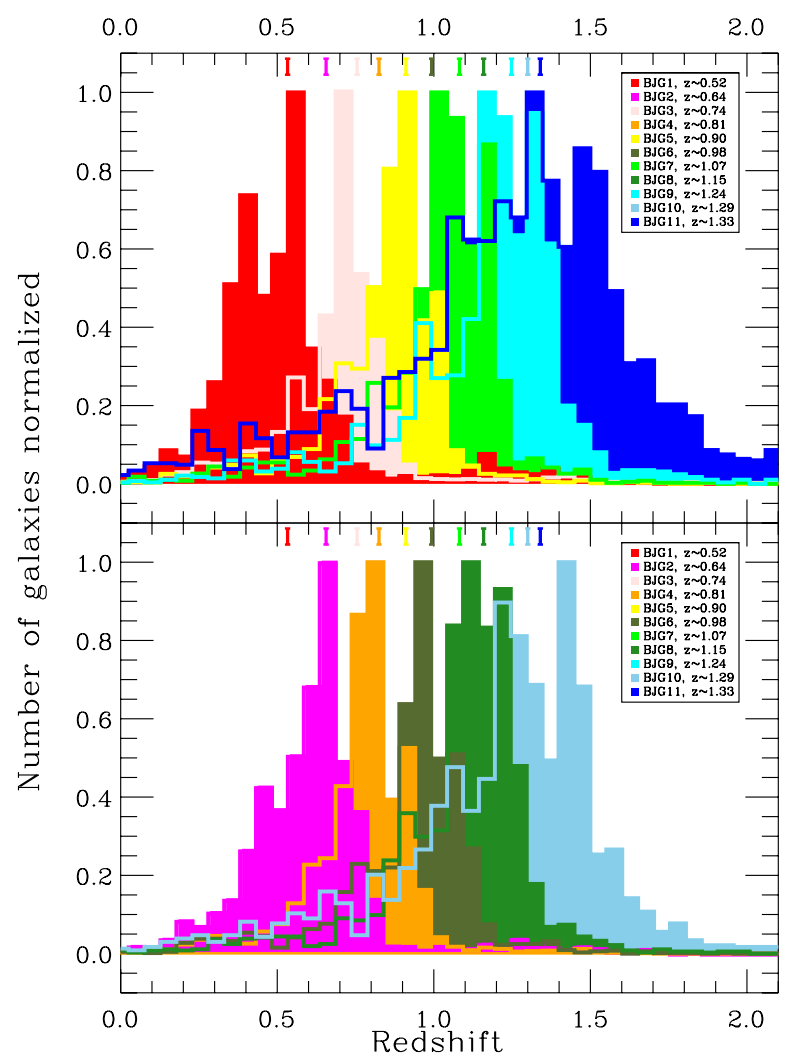

Fig. 4. Photometric redshift distribution of the selected samples. Upper panel: red, pink, yellow, light green, cyan, and blue histograms show the $z \mathrm{BPZ}$ distribution of the $B J G_{1}, B J G_{3}, B J G_{5}, B J G_{7}, B J G_{9}$, and $B J G_{11}$ samples, respectively. Lower panel: magenta, orange, dark green, green, and light blue histograms show the $z \mathrm{BPZ}$ distribution for the $B J G_{2}, B J G_{4}, B J G_{6}, B J G_{8}$, and $B J G_{10}$ samples, respectively. The vertical colored lines show the median of the $z \mathrm{BPZ}$ distribution for the selected samples. The label locate in the upper-right corner reports the median redshift.

better visualize the distribution tails, the upper panel shows the BPZ distribution of the $B J G_{1}, B J G_{3}, B J G_{5}, B J G_{7}, B J G_{9}$, and $B J G_{11}$ samples, while the lower panel presents the
Table 3. Median physical properties of the $B J G$ samples.

\begin{tabular}{cccccc}
\hline \hline Name & $N$ & $z \mathrm{BPZ}$ & $\begin{array}{c}\left\langle M_{*}\right\rangle \\
M_{\odot}\end{array}$ & $\begin{array}{c}S F R \\
M_{\odot} \mathrm{yr}^{-1}\end{array}$ & $\begin{array}{c}\text { Age } \\
{[\mathrm{Gyr}]}\end{array}$ \\
\hline$B J G_{1}$ & 5489 & $0.52_{-0.14}^{+0.16}$ & $10.15_{-0.13}^{+0.11}$ & $0.08_{-0.23}^{+0.22}$ & $5.52_{-1.87}^{+1.62}$ \\
$B J G_{2}$ & 5174 & $0.64_{-0.18}^{+0.11}$ & $10.25_{-0.13}^{+0.12}$ & $0.27_{-0.22}^{+0.23}$ & $5.13_{-1.76}^{+1.50}$ \\
$B J G_{3}$ & 4497 & $0.74_{-0.17}^{+0.10}$ & $10.30_{-0.14}^{+0.13}$ & $0.38_{-0.24}^{+0.23}$ & $4.83_{-1.68}^{+1.40}$ \\
$B J G_{4}$ & 4012 & $0.81_{-0.14}^{+0.12}$ & $10.37_{-0.15}^{+0.13}$ & $0.42_{-0.26}^{+0.25}$ & $4.67_{-1.62}^{+1.29}$ \\
$B J G_{5}$ & 3550 & $0.90_{-0.18}^{+0.12}$ & $10.36_{-0.16}^{+0.14}$ & $0.49_{-0.25}^{+0.24}$ & $4.37_{-1.54}^{+1.24}$ \\
$B J G_{6}$ & 2878 & $0.98_{-0.19}^{+0.12}$ & $10.41_{-0.17}^{+0.15}$ & $0.52_{-0.27}^{+0.25}$ & $4.12_{-1.46}^{+1.15}$ \\
$B J G_{7}$ & 2231 & $1.07_{-0.20}^{+0.12}$ & $10.46_{-0.17}^{+0.15}$ & $0.63_{-0.26}^{+0.25}$ & $3.83_{-1.40}^{+1.09}$ \\
$B J G_{8}$ & 2325 & $1.15_{-0.21}^{+0.12}$ & $10.45_{-0.19}^{+0.16}$ & $0.69_{-0.27}^{+0.26}$ & $3.63_{-1.36}^{+1.05}$ \\
$B J G_{9}$ & 2058 & $1.24_{-0.25}^{+0.14}$ & $10.45_{-0.20}^{+0.18}$ & $0.72_{-0.25}^{+0.25}$ & $3.41_{-1.32}^{+1.01}$ \\
$B J G_{10}$ & 2140 & $1.29_{-0.33}^{+0.21}$ & $10.38_{-0.23}^{+0.19}$ & $0.72_{-0.22}^{+0.24}$ & $3.16_{-1.29}^{+1.05}$ \\
$B J G_{11}$ & 3391 & $1.33_{-0.42}^{+0.28}$ & $10.34_{-0.24}^{+0.20}$ & $0.72_{-0.21}^{+0.23}$ & $2.99_{-1.27}^{+1.05}$ \\
\hline
\end{tabular}

Notes. Column 1, name of the selected sample; Col. 2, number of galaxies selected; Col. 3, BPZ photometric redshift; Col. 4, logarithm of the stellar mass (Chabrier IMF); Col. 5, logarithm of the star formation rate; Col. 6, galaxy age.

$B J G_{2}, B J G_{4}, B J G_{6}, B J G_{8}$, and $B J G_{10}$ samples. The median of zBPZ distribution, reported in Table 3, clearly agree with the median of the expected redshift range estimated through the twocolor selection criteria reported in Table 2.

\subsection{SED fitting}

For each galaxy of the $B J G$ samples, we fit the 20 optical bands plus the three NIR bands using the Bayesian SED modeling code iSEDfit (Moustakas et al. 2013). After we fixed the redshift to the best-fit value given by BPZ in the ALHAMBRA cata$\log$ (Molino et al. 2014), iSEDfit calculated the marginalized posterior probability distributions for the physical parameters in a certain model space that was previously defined. Using a Monte Carlo technique, we generated 20,000 model SEDs with delayed star formation histories SFH $\sim t e^{-t / \tau}$, where $\tau$ is the star formation timescale. The SEDs were computed employing the flexible stellar population synthesis models (FSPS, v 2.4; Conroy et al. 2009; Conroy \& Gunn 2010) that are based on the MILES (Sanchez-Blázquez et al. 2006) and Basel (Lejeune et al. 1997, 1998; Westera et al. 2002) stellar libraries. We assumed a Chabrier (2003) initial mass function from $0.1-100 M_{\odot}$ and the time-dependent attenuation curve of Charlot \& Fall (2000). We adopted uniform priors on stellar metallicity $Z / Z_{\odot} \in[0.04,1.0]$, galaxy age $t \in\left[0.01\right.$, age $\left.\left(z_{\mathrm{BPZ}}\right)\right] \mathrm{Gyr}$, rest-frame $V$-band attenuation $A_{V} \in[0-3] \mathrm{mag}$, and star formation timescale $\tau \in$ $\left[0.01, \operatorname{age}\left(z_{\mathrm{BPZ}}\right)\right] \mathrm{Gyr}$, where $\operatorname{age}\left(z_{\mathrm{BPZ}}\right)$ is the age of the Universe at each galaxy's photometric redshift.

Figures 9 and 10 show the SEDs of a galaxy randomly picked from each $B J G$ sample. The green filled symbols show the data and their error bar. The model that minimize the $\chi^{2}$ is drawn with the red line. The universe of models, generated using the aforementioned setup, and scaled by their reduced $\chi^{2}$ is shown with the blue shading.

For each $B J G$ sample and certain physical properties, the median value of the sum of the posterior probability distributions were calculated and are reported in Table 3. The uncertainties indicate the $1 \sigma$ confidence level; to account for asymmetric distributions, we determined the percentiles 16 and 84 . 
P. Troncoso Iribarren et al.: The evolution of Balmer jump selected galaxies in the ALHAMBRA survey

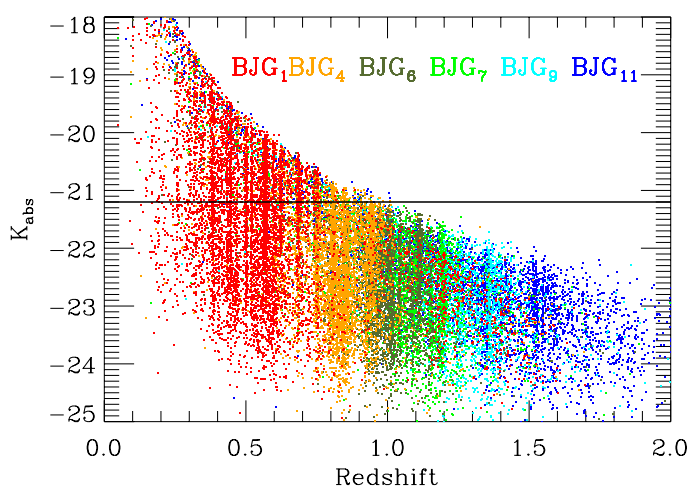

Fig. 5. Absolute magnitude as a function of redshift. The colored dots represent a galaxy of each $B J G$ sample, using the same color code than in Fig. 4. The horizontal solid line shows the absolute magnitude cut $K_{\mathrm{abs}}=-21.2$.

\subsection{Comparison between BJG samples}

In this subsection, we compare the properties derived from SED fitting of the $B J G$ samples for galaxies of similar $K$-band absolute magnitude, i.e. similar stellar mass. Therefore, in addition to restricting the $B J G$ samples within the magnitude survey limit (see Sect. 3), we applied an absolute magnitude limit for all samples. In the following, we determine the most appropriate absolute magnitude limit that allows us to build these samples. Figure 5 shows the $K$-band absolute magnitude, obtained through the iSEDfit, as a function of BPZ redshift. The red, orange, green, cyan, light blue, and blue dots indicate the galaxies in the $B J G_{1}, B J G_{4}, B J G_{6}, B J G_{7}, B J G_{9}$, and $B J G_{11}$ samples with apparent magnitude brighter than the magnitude survey limit $K=22$, respectively. We can note that partly as a result of the Malquist bias, the farthest sample $B J G_{11}$ is roughly complete only until $K_{\mathrm{abs}}=-22.5$ (blue dots). However, by choosing this bright absolute limit to compare all $B J G$ samples, we restrict our study to the most massive and bright objects and also enormously reduce the statistics for each sample, especially at low redshift. Hence, we decided to study all objects brighter than $K_{\mathrm{abs}}=-21.2$, which corresponds to the absolute limit where the $B J G_{5}$ sample at $z \sim 1$ is complete and comparable, in terms of $K_{\text {abs }}$ luminosity, to the other lower redshift $B J G_{n<6}$ samples. In Fig. 5 the black solid line shows the absolute magnitude limit $K_{\text {abs }}=-21.2$. We chose the $z \sim 1$ limit because the ALHAMBRA Gold data are an $F 814 W$ (i.e., almost an $I$-band) selected catalog and thus are less sensitive to galaxies at $z \geq 1$ with a pronounced Balmer jump. For galaxies at $z \geq 1$, the spectral region directly blueward of the Balmer jump is barely or not at all detected in the $F 814 \mathrm{~W}$ because the Balmer jump falls in the $I_{13}$ band and the region directly blueward of it falls in the $R_{12}$ band. Since the $F 814 W$ image detection is a linear combination involving the $R_{12}$ band, these types of galaxies are barely or not at all detected as sources in the final catalog. We expect a deeper selection of galaxies with a pronounced Balmer jump at $z<1$. Hence, the $B J G$ samples at $z \leqslant 1$ selected from $X_{n \leq 13}$ bands are optimized to be complete according to the $F 814 \mathrm{~W}$ selection, whereas the samples at $z \geq 1$ are incomplete. The level of incompleteness of the $B J G_{n \geq 6}$ samples is difficult to quantify because there may be many effects working together (e.g., the $F 814 W$ band is a linear combination of ALHAMBRA bands and blueward regions of the Balmer jump that are undetectable as a result of intrinsic galaxy properties such as redshift and dust excess). To minimize the incompleteness of our samples in the comparison of the physical properties at different redshifts, we
Table 4. Median physical properties of the $B J G K$ samples considering the absolute magnitude limit $K=-21.2$.

\begin{tabular}{lccccc}
\hline \hline Name & $N$ & $z \mathrm{BPZ}$ & $\begin{array}{c}\left\langle M_{*}\right\rangle \\
M_{\odot}\end{array}$ & $\begin{array}{c}S F R \\
M_{\odot} \mathrm{yr}^{-1}\end{array}$ & $\begin{array}{c}\text { Age } \\
{[\mathrm{Gyr}]}\end{array}$ \\
\hline$B J G K_{1}$ & 3322 & $0.56_{-0.14}^{+0.18}$ & $10.50_{-0.11}^{+0.10}$ & $0.24_{-0.30}^{+0.29}$ & $5.80_{-1.87}^{+1.43}$ \\
$B J G K_{2}$ & 3778 & $0.67_{-0.16}^{+0.11}$ & $10.46_{-0.12}^{+0.11}$ & $0.40_{-0.26}^{+0.27}$ & $5.31_{-1.74}^{+1.37}$ \\
$B J G K_{3}$ & 3576 & $0.75_{-0.12}^{+0.10}$ & $10.45_{-0.13}^{+0.12}$ & $0.46_{-0.26}^{+0.26}$ & $4.92_{-1.67}^{+1.31}$ \\
$B J G K_{4}$ & 3480 & $0.83_{-0.09}^{+0.11}$ & $10.45_{-0.14}^{+0.13}$ & $0.49_{-0.28}^{+0.27}$ & $4.69_{-1.60}^{+1.24}$ \\
$B J G K_{5}$ & 3150 & $0.92_{-0.12}^{+0.10}$ & $10.44_{-0.15}^{+0.14}$ & $0.55_{-0.26}^{+0.26}$ & $4.37_{-1.52}^{+1.19}$ \\
\hline
\end{tabular}

Notes. Column 1, name of the selected sample; Col. 2, number of galaxies selected from the $B J G$ samples after the $K$-band absolute cut; Col. 3, BPZ photometric redshift; Col. 4, stellar mass (Chabrier IMF); Col. 5, star formation rate; Col. 6, galaxy age.

redefine the first five $B J G$ samples in the following by selecting all galaxies brighter than $K_{\mathrm{abs}}=-21.2$. Furthermore, the $B J G_{n}$ samples with $n \geq 6$ are not considered in the following analysis. Table 4 presents the properties of the final sample definition $\left(B J G K_{n}\right.$, with $\left.n<6\right)$, which takes into account the absolute luminosity cut $\left(K_{\mathrm{abs}}=-21.2\right)$. In Fig. 6 the comparison of the physical properties of galaxies with similar $K$-band luminosity is shown. Red squares show the median value of the probability distribution for each $B J G K_{n}$ sample (with $n<6$ ), while the colored areas indicate their dispersion. The error bars show the median error of the properties derived through the iSEDfit. In Sect. 6.3 these results are discussed.

\section{Halo masses through clustering analysis}

In hierarchical clustering, structures build up in time from small density fluctuations. Small structures agglomerate to build large structures. Dark matter halos are biased tracers of the underlying matter density field. Massive halos lie in higher and rarer density peaks and are more clustered than lower mass halos. If a galaxy population is hosted by halos of a given mass, then the clustering amplitude of the galaxy population, compared to the expected dark matter clustering at the same redshift, can be used to derive the typical halo mass corresponding to that galaxy population. This is encapsulated in the bias parameter, $b$, defined as $b^{2}=\xi_{\text {gal }}(r) / \xi_{\mathrm{DM}}(r)$, where $\xi_{\text {gal }}$ and $\xi_{\mathrm{DM}}$ are the two-point spatial correlation functions for galaxies and dark matter, respectively. The correlation function at a certain redshift, $\xi(r, z)$, can be characterized with a power law and a correlation length, $r_{0}$, through $\xi(r, z)=\left(r / r_{0}(z)\right)^{\gamma}$, where $\gamma$ is the power index. For sparse samples and at small scales this representation is typically used. In larger surveys and simulations the correlation function is usually modeled by combining terms for galaxies in the same halo and in different ones (Zehavi et al. 2011; Contreras et al. 2013).

In the following, we measure the bias and masses of the halos that host the $B J G K_{n<6}$ samples. Specifically, for each $B J G K_{n<6}$ sample, we calculate the two-point angular correlation function, and then, using the Limbers deprojection, given a redshift distribution and a cosmological model, we calculate the correlation length. The bias parameter follows from $r_{0}$ through $b^{2} \simeq \sigma_{8, \text { gal }}^{2}\left(r_{0}, z\right) / \sigma_{8, \mathrm{DM}}^{2}(z)$, where $\sigma_{8 \text {,gal }}\left(\sigma_{8, \mathrm{DM}}\right)$ is the root mean square fluctuation amplitude in $8 \mathrm{~h}^{-1} \mathrm{Mpc}$ spheres of galaxies (dark matter). The halo mass is obtained from the bias by using the models of Sheth et al. (2001). We chose this procedure and the $8 h^{-1} \mathrm{Mpc}$ scale to directly compare our results with previous works that connected progenitors and descendants of 

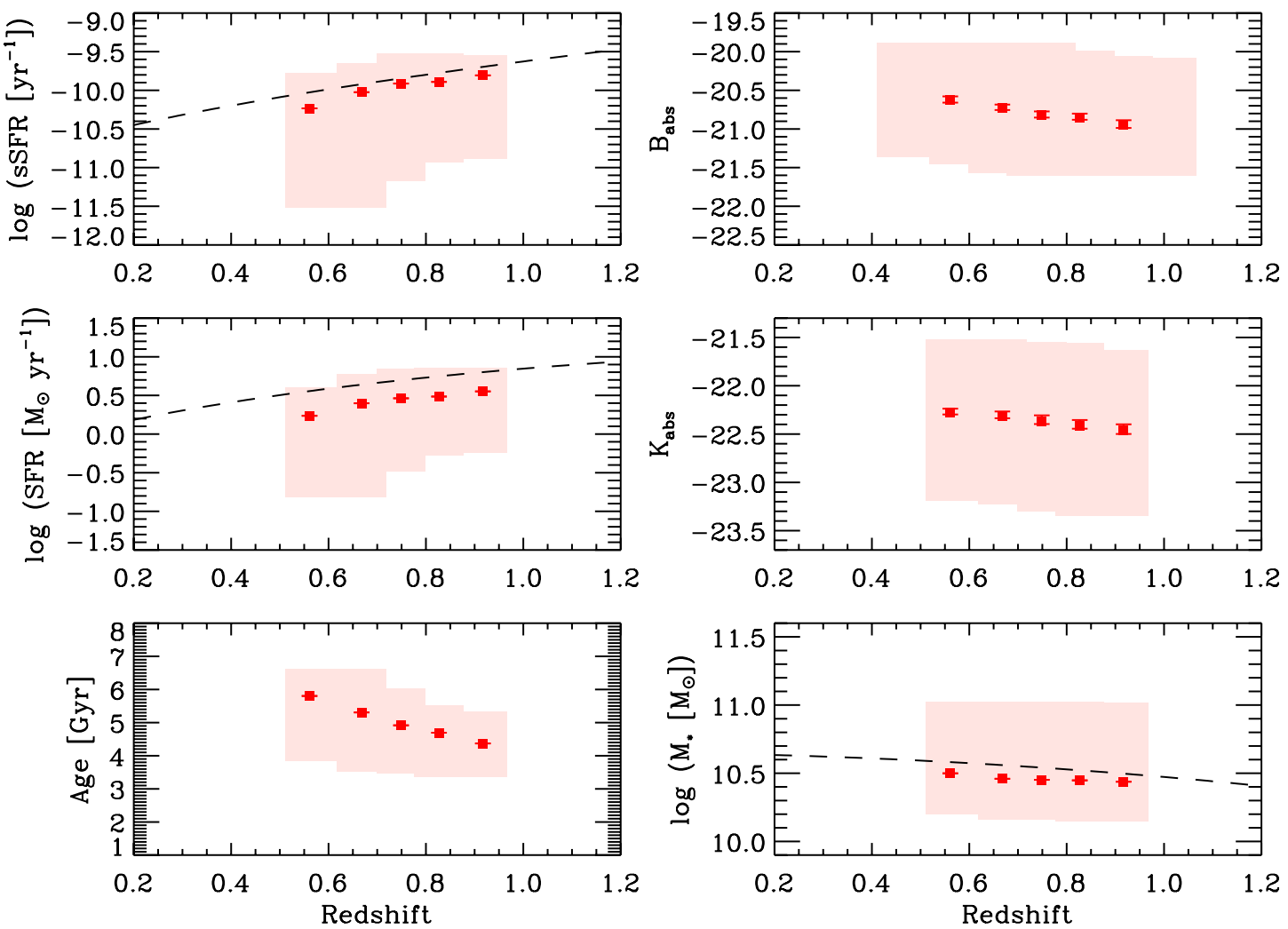

Fig. 6. Evolution of the physical properties of the $B J G K_{n<6}$ samples. The panels show the median of the probability distribution of (top to bottom and left to right) the specific star formation rate, star formation rate, age, $B$-band luminosity, $K$-band luminosity, and stellar mass. Red squares show the evolution of $B J G K_{n<6}$ galaxies brighter than $K=-21.2$. The colored areas represent the dispersion of each sample. The error bars correspond to the median error of the physical properties determined by the iSEDfit. Dashed lines show the fit to the CANDELS analysis taken from van Dokkum et al. (2013) for galaxies of stellar masses similar to the Milky Way.

samples of star-forming galaxies (see Fig. 7, Adelberger et al. 2005; Ouchi et al. 2005; Kashikawa et al. 2006; Gawiser et al. 2007; Hildebrandt et al. 2007; Hayashi et al. 2007; Blanc et al. 2008; Hartley et al. 2008; Yoshida et al. 2008; Guaita et al. 2010; McCracken et al. 2010; Lin et al. 2012). More detailed treatments, such as considering both halo terms and different scales, are beyond the scope of this paper.

\subsection{Angular correlation and correlation length}

To determine the angular correlation function, we used the Landy and Szalay (1993) prescription

$w(\theta)=\left(N_{g g}-2 N_{g r}+N_{r r}\right) / N_{r r}$

where $N_{g g}$ and $N_{r r}$ are the number of pairs at a separation $\theta$ of galaxies in the catalog and points in a random catalog with the same layout as the galaxy sample, respectively. $N_{g r}$ is the cross number of points between the galaxy and random distributions. For our calculations we considered five ALHAMBRA fields (see Sect. 2) that encompass 36 pointing catalogs. Errors were estimated by a jackknife method, which has been shown to be a robust error estimator (Zehavi et al. 2011; Cabré et al. 2007), although it can overestimate the variance on small scales (Norberg et al. 2009). We calculated the angular correlation function 36 times, each time eliminating one catalog out of the 36 available. The uncertainty was estimated as the variance of $w(\theta)$. We followed the method of Infante (1994), Quadri et al. (2007) to correct for the integral constraint. As mentioned above, to calculate $r_{0}$ we used the power-law approximation $\xi(r, z)=$ $\left(r(z) / r_{0}(z)\right)^{-\gamma}$ that depends on the spatial separation and redshift. Likewise, the two-point angular function is $w(\theta, z)=A_{w}(z) \theta^{(1-\gamma)}$, where $A_{w}(z)$ is the angular amplitude. By fitting the correlation function to this power law, between 0.005 and 0.2 degree, the $A_{w}(z)$ is inferred. The angular amplitudes are reported in Table 5. To calculate the spatial from the angular function, we used the Limber (1953) inversion, which requires a redshift distribution $N(z)$, and assumed a cosmological model. Limber's inversion involves solving the following integral (Kovac et al. 2007):

$r_{0}^{\gamma}=\frac{A_{w}\left(c / H_{0}\right)\left[\int N(z) \mathrm{d} z\right]^{2}}{C_{\gamma} \int F(z) D_{\theta}^{1-\gamma}(z) N^{2}(z) g_{d}(z) \mathrm{d} z}$,

where the cosmology plays a role in the Hubble parameter $H_{0}$, $g_{d}(z)=(1+z)^{2} \sqrt{1+\Omega_{\mathrm{m}} z+\Omega_{\Lambda}\left[(1+z)^{-2}-1\right]}$, and in the angular diameter distance $D_{\theta}$. The parameter $C_{\gamma}$ depends on the power index such that $C_{\gamma}=\Gamma(0.5) \frac{\Gamma[0.5(\gamma-1)]}{\Gamma[0.5 \gamma]}$. To be consistent internally and compare our results with other works (Adelberger et al. 2005; Ouchi et al. 2005; Kashikawa et al. 2006; Hayashi et al. 2007; Hildebrandt et al. 2007; Gawiser et al. 2007; Blanc et al. 2008; Hartley et al. 2008; Yoshida et al. 2008; Guaita et al. 2010; McCracken et al. 2010; Lin et al. 2012), the value of $\gamma$ was fixed to the canonical $\gamma=1.8$. This value is fully justified by experiments where $\gamma$ was left free and is consistent with the clustering of luminosity-selected ALHAMBRA samples (Arnalte-Mur et al. 2014). $F(z)$ accounts for the redshift evolution of the correlation function, where $F(z)=(1+z)^{-(3+\epsilon)}$, and we used $\epsilon=-1.2$, 


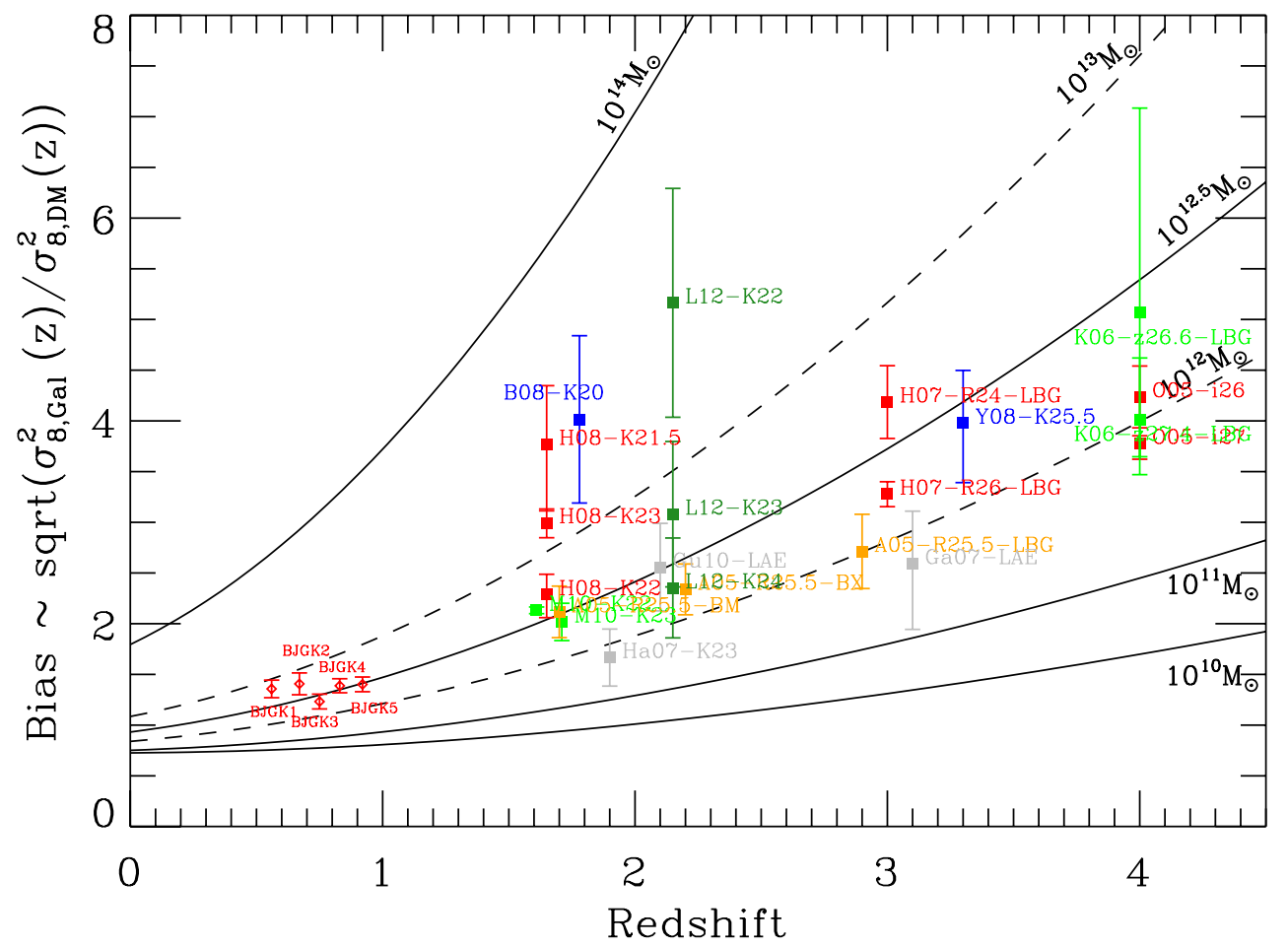

Fig. 7. Evolution of the bias factor, calculated with the variance at $8 h^{-1} \mathrm{Mpc}$, for samples of star-forming galaxies. Red diamonds indicate the $B J G K_{n<6}$ samples. At $z \sim 2$, the gray, blue, red, green, and light green squares show the $s B z K$ samples of Hayashi et al. (2007), Blanc et al. (2008), Hartley et al. (2008), Lin et al. (2012), and McCracken et al. (2010), respectively. Yellow squares show the $B M$ and $B X$ selected galaxies of Adelberger et al. (2005). At $z \sim 3$, the blue, green, and red squares indicate the $L B G$ s selected by Yoshida et al. (2008), Kashikawa et al. (2006), Hildebrandt et al. (2007), Ouchi et al. (2005), respectively. Gray squares show the Ly- $\alpha$ emitters selected by Guaita et al. (2010), Gawiser et al. (2007). The lines show the bias evolution for different halo masses (Sheth et al. 2001), which are indicated below each line at the right side of the panel.

Table 5. Clustering properties of the $B J G K$ samples.

\begin{tabular}{lccccccc}
\hline \hline Name & $N_{\text {gal }}$ & $\begin{array}{c}\langle z\rangle \\
\mathrm{BPZ}\end{array}$ & $\begin{array}{c}\left\langle K_{\text {abs }}\right\rangle \\
\mathrm{mag}\end{array}$ & $\begin{array}{c}A_{w} \\
10^{-3}\end{array}$ & $\begin{array}{c}r_{0} \\
{\left[h^{-1} \mathrm{Mpc}\right]}\end{array}$ & $\begin{array}{c}\text { Bias } \\
\left(r=8 h^{-1} \mathrm{Mpc}\right)\end{array}$ & $\begin{array}{c}\log M_{\mathrm{h}} \\
{\left[h^{-1} M_{\odot}\right]}\end{array}$ \\
\hline$B J G K_{1}$ & 3332 & $0.56_{-0.1}^{+0.2}$ & -22.3 & $4.2 \pm 0.2$ & $4.67 \pm 0.33$ & $1.36 \pm 0.09$ & $12.71_{-0.15}^{+0.13}$ \\
$B J G K_{2}$ & 3778 & $0.67_{-0.2}^{+0.1}$ & -22.3 & $4.3 \pm 0.5$ & $4.58 \pm 0.39$ & $1.41 \pm 0.11$ & $12.66_{-0.17}^{+0.15}$ \\
$B J G K_{3}$ & 3576 & $0.75_{-0.1}^{+0.1}$ & -22.4 & $4.0 \pm 0.2$ & $3.79 \pm 0.25$ & $1.24 \pm 0.07$ & $12.27_{-0.16}^{+0.14}$ \\
$B J G K_{4}$ & 3480 & $0.83_{-0.1}^{+0.1}$ & -22.4 & $4.2 \pm 0.1$ & $4.15 \pm 0.23$ & $1.40 \pm 0.07$ & $12.45_{-0.12}^{+0.10}$ \\
$B J G K_{5}$ & 3150 & $0.92_{-0.1}^{+0.1}$ & -22.5 & $3.7 \pm 0.2$ & $4.01 \pm 0.23$ & $1.40 \pm 0.07$ & $12.37_{-0.12}^{+0.11}$ \\
\hline
\end{tabular}

Notes. Column 1, name of the selected sample; Col. 2, number of galaxies selected from the $B J G$ samples after the $K$-band absolute cut and image mask; Col. 3, BPZ photometric redshift; Col. 4, medium $K$-band absolute magnitude; Col. 5, amplitude of correlation; Col. 6, correlation length; Col. 7, bias factor calculated with the variance at $8 h^{-1} \mathrm{Mpc}$. Column 8 , logarithm of the halo mass in units of $\left[h^{-1} M_{\odot}\right]$.

which corresponds to the value adopted for a constant clustering in comoving coordinates (Quadri et al. 2007). To calculate the correlation length error, we took the error contribution from the amplitude of the angular correlation function, the Poissonian errors $(\sim \sqrt{N(z)})$, and the effects of the photometric redshift error in shifting and broadening $N(z)$ into account.

\subsection{Bias and mass measurements}

In turn we estimated the bias parameter, $b$, from the correlation length. As pointed out above, $b$ is related to $r_{0}$ through the spatial correlation function by $b^{2} \approx \xi_{\text {gal }}(r, z) / \xi_{\mathrm{DM}}(r, z)$ or $b^{2} \approx \sigma_{\mathrm{gal}}^{2}(r, z) / \sigma_{\mathrm{DM}}^{2}(r, z)$. The numerator $\xi_{\mathrm{gal}}(r, z) \approx J_{2} \sigma_{\mathrm{gal}}^{2}(r, z)$ was taken from Peebles (1980) (Eq. (59.3)), where $J_{2}$ is a parameter defined in terms of $\gamma$ as $J_{2}=72 /[(3-\gamma)(4-$ $\left.\gamma)(6-\gamma) 2^{\gamma}\right]$. We fixed the spatial scale at $8 h^{-1} \mathrm{Mpc}$ such that $\xi_{\text {gal }}(8, z)=\left(r_{0}(z) / 8 h^{-1} \mathrm{Mpc}\right)^{\gamma}$. The evolution of the dark matter density variance in a comoving sphere of radius $8 h^{-1} \mathrm{Mpc}$ is $\sigma_{\mathrm{DM}}^{2}(8, z)=\sigma_{8} D(z)$, where $D(z)$ is the linear growth factor at redshift $\mathrm{z}$. The bias measured for each $B J G K$ sample at a scale of $8 h^{-1} \mathrm{Mpc}$ is reported in Table 5 .

Figure 7 shows the bias factor as a function of redshift for the BJGK samples (red diamonds). The lines show the bias evolution for different halo masses (Sheth et al. 2001), which are indicated below each line at the right side of the panel. The BJGK samples approximately follows the bias evolution of halos of 


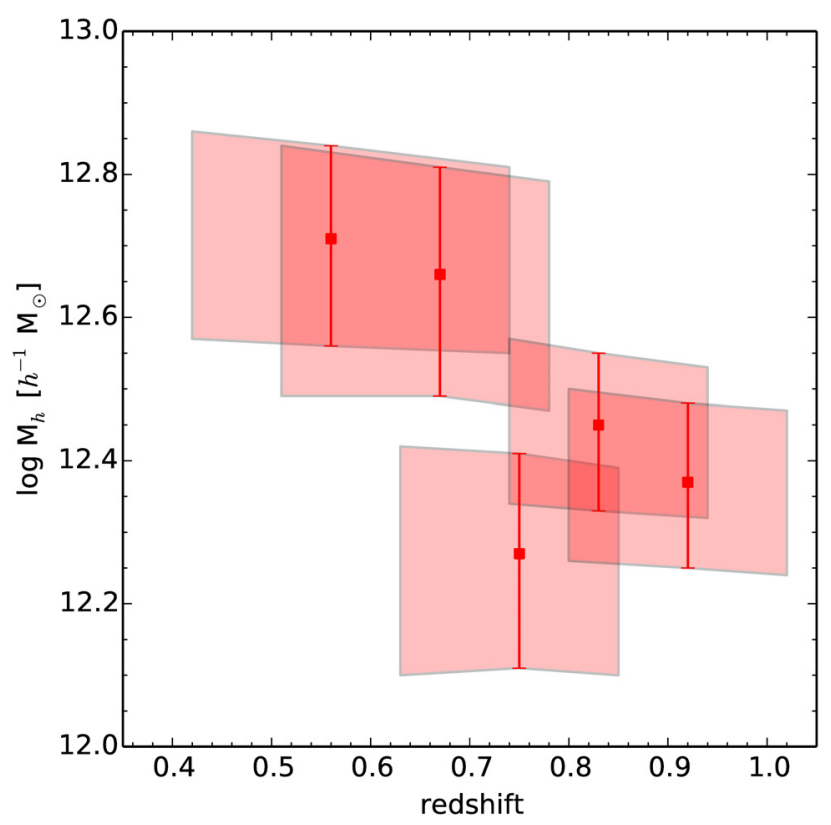

Fig. 8. Halo mass as function of redshift for the $B J G K_{n<6}$ samples. Squares show the median values of the halo mass, while polygons draw the mass limit taking into account the error of redshift and bias.

masses $\sim 10^{12.5} h^{-1} M_{\odot}$. Finally, we calculated the halo mass using Eq. (8) of Sheth et al. (2001), which relates the bias with the peak height, $v=\delta_{\mathrm{sc}}(z) / \sigma(M, z)$, where $\delta_{\mathrm{sc}}$ is the critical overdensity computed using the spherical collapse model. Here we assumed $\delta_{\mathrm{sc}}=1.69$. Then, the halo mass $M_{\mathrm{h}}$ was obtained through $\sigma(M, z)$ evaluated at the redshifts of the $B J G K$ samples listed in Table 4. In Fig. 8 the halo mass of the BJGK samples as a function of redshift is shown. This is calculated with the variance at $8 h^{-1} \mathrm{Mpc}$. Squares show the median values of the halo mass, while polygons show a conservative limit. This draws the mass limits considering the $1 \sigma$ deviation of the redshift and bias. The results are presented in Table 5.

\section{Discussion}

\subsection{Selection technique}

The new two-color selection technique $U_{2} X_{n} K$ based on ALHAMBRA medium-bands allows us to extract eleven samples of star-forming galaxies at $z>0.5$ in narrower redshift ranges than was possible in previous studies (Daddi et al. 2004; Steidel et al. 2004; Adelberger et al. 2005). This selection method is based purely on the ALHAMBRA photometric data and does not depend on the models assumptions, method, or templates used to determine the photometric redshifts or properties derived from SED fit. We validated this technique with the Bruzual \& Charlot (2003) models and also checked other stellar population synthesis models, which give consistent results.

The ALHAMBRA GOLD data are an $F 814 W$-selected cata$\log$, thus it is less sensitive to the regions blueward of the Balmer jump of galaxies at $z>1$. The $R_{12}$ band only contributes to $10 \%$ of the final $F 814 \mathrm{~W}$ detection image, while the $I_{13}$, and $I_{14}$ bands contribute $18 \%$ each. Hence, the detection in the $F 814 W$ image becomes poorer for galaxies at $z \geq 1$ with a pronounced Balmer jump.

To avoid contamination of low-redshift galaxies in our samples, we used the bluest band available to sample the region blueward of the Balmer jump. Hence, we studied the detection level of the $U_{1}$ and $U_{2}$, which reaches $97 \%$ and $99.7 \%$, respectively. Since $U_{2}$ has a higher detection level than $U_{1}$, we tailored the selection technique using the $U_{2}$ band.

To select samples at $z<0.5$, we tried to use filters bluer than the $R_{9}$, whose central wavelength is lower than $613.5 \AA$. Nevertheless, the theoretical evolution of the color $U_{2} X_{n<9} K$ of passive and star-forming galaxies, based on the Bruzual \& Charlot (2003) models, tends to occupy the same locus in the color-redshift $\left(U_{2} X_{n<9} K-z\right)$ plane. Hence, it does not allow separating the star-forming from the passive galaxies as clearly as for the $U_{2} X_{n} K$ selection with $n>9$ (see Figs. 2 and 3 ).

The number density of each $B J G$ sample decreases with redshift, probably because of the nature of these objects or because of the Malquist-bias selection effect in the $U_{2}$-band, which we did not consider here. In addition, we verified our selection method with the BPZ photometric redshifts, whose uncertainty increases with redshift, and therefore the number densities might be underestimated according to $\delta_{z}(B P Z)=0.014 z(B P Z)$.

Based on this color technique (visual inspection of Figs. 2 and 3), the redshift distributions should be narrower than in Fig. 4. The distributions can become wider if the errors of the photometric redshifts are properly taken into account. The mean formal BPZ errors of the $B J G_{1}$ and $B J G_{11}$ samples are 0.02 and 0.03 , which corresponds to $20 \%$ and $30 \%$ of the total expected width $(\leq 0.1)$, respectively. We performed simulations considering Gaussian redshift distributions filled randomly using the same number of galaxies found in each sample, the expected width $(0.1)$, and the formal $\mathrm{BPZ}$ error $\delta_{z}(B P Z)=$ $0.014(1+z(B P Z))$. By perturbing the photometric redshift with its corresponding error and randomly choosing a positive or negative variance, the width of the distributions increases by a factor of 1.5 for the first $B J G_{1}$ sample and up to twice this value for the $B J G_{11}$ sample with increasing redshift. Clearly, higher photometric redshift errors increase the distribution width. In the AHAMBRA data, BPZ tends to lower precision at $I<24.5 \mathrm{AB}$ (Molino et al. 2014). The global redshift distribution shows a mean of $\langle z\rangle=0.86$ at this magnitude limit. Hence the distributions at $z \geq 0.9$ tend to broaden even more because the photometric redshift errors exceed $0.014(1+z)$. For a distribution at $z \sim 1$ with an original width of 0.1 and $\delta_{z}(B P Z)=0.03(1+z(B P Z))$, the width increases up to three times. Outliers that are due to either photometric redshift or the color selection technique can also contribute to the tails and may also broaden the photometric redshift distributions.

We also investigated the selection method by creating an $X_{n}$ band composed of two, three, and four consecutive ALHAMBRA bands. This increased the statistics, accuracy, and width of the redshift distribution of each sample. Nevertheless, to fully exploit the capabilities of a multi-medium band survey in selecting galaxies in small redshift ranges, we chose to use a unique band in our selection method.

Viironen et al. (2015) have shown that is possible to obtain a clean galaxy sample using the redshift probability distributions, which is by definition (intrinsically) not complete. The fact of choosing a certain probability threshold implies that some galaxies will not be considered in the final sample. To overcome the problem of purity versus completeness, they suggested using the information of the whole $z \mathrm{PDF}$ to select each sample. In this work we tailored and used a two-color criterion that considered all the observational data available, selecting a complete sample, but at the same time was probably more contaminated than the samples selected according to redshift probability distributions. We used the $z \mathrm{BPZ}-B J G$ distributions to calculate this contamination by estimating how many objects have a BPZ redshift 
P. Troncoso Iribarren et al.: The evolution of Balmer jump selected galaxies in the ALHAMBRA survey

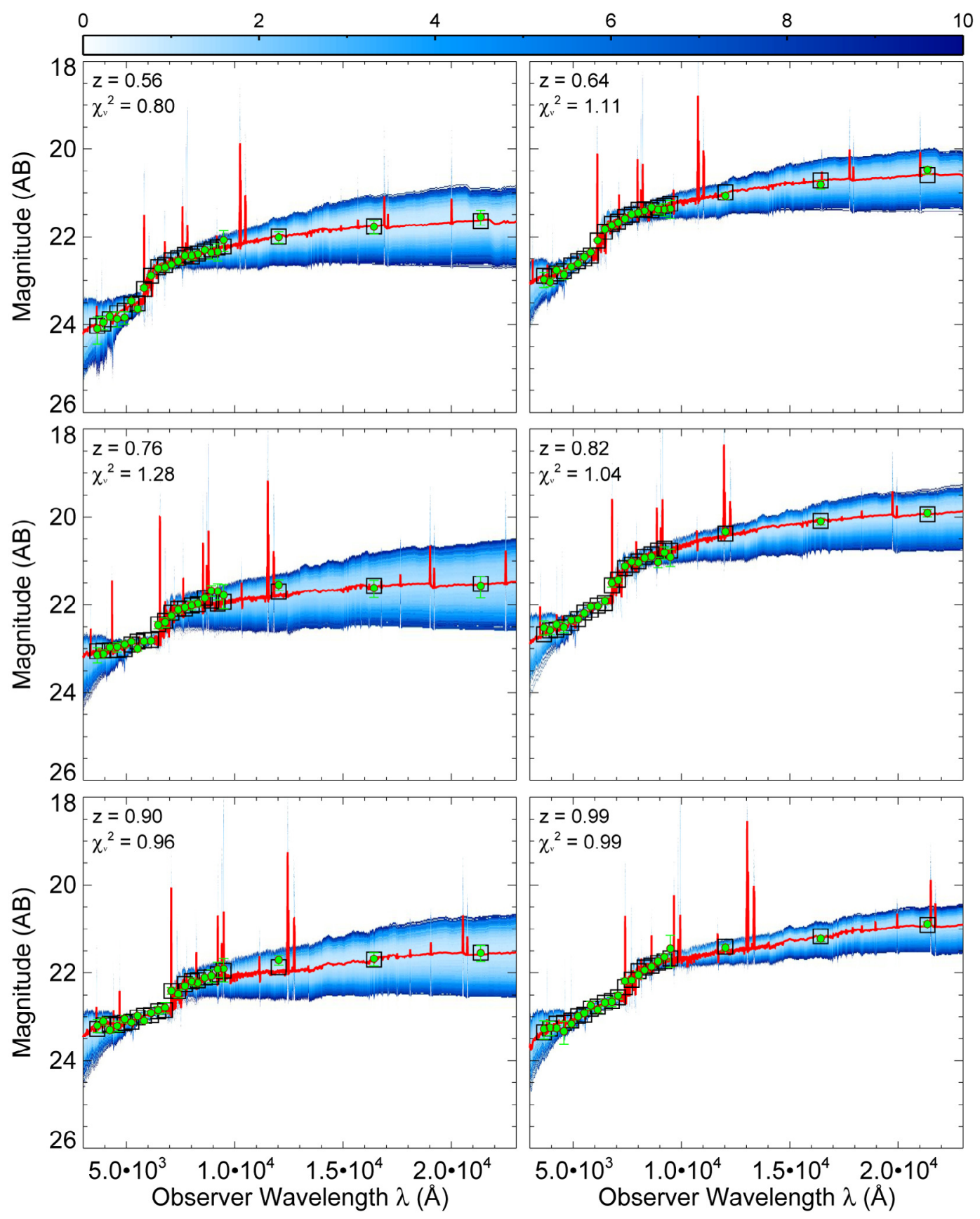

Fig. 9. Spectral energy distribution of a star-forming galaxy in the ALHAMBRA survey. From left to right and top to bottom, the panel shows the SED of a galaxy randomly picked from the $B J G_{1}, B J G_{2}, B J G_{3}, B J G_{4}, B J G_{5}$, and $B J G_{6}$ samples. The filled green dots show the ALHAMBRA photometric data. The red line shows the model that minimizes the $\chi^{2}$ found by iSEDfit, the minimum reduced $\chi^{2}$ is indicated in the upper left corner, in addition to the BPZ photometric redshift. The black squares mark the ALHAMBRA photometry of the best model. The blue shading shows the universe of models, generated by iSEDfit (see Sect. 4.2), scaled by their reduced $\chi^{2}$. The color bar indicates the reduced $\chi^{2}$ scale.

lower or higher than $3 \sigma$ of its corresponding redshift distribution. Thus, it encompasses from $1 \%$ to $15 \%$ of each sample depending on redshift. Specifically, $1 \%, 2 \%, 2 \%, 3 \%, 4 \%$, $5 \%, 6 \%, 7 \%, 8 \%, 10 \%, 12 \%$, and $15 \%$ of the sample $B J G_{1}$, $B J G_{2}, B J G_{3}, B J G_{4}, B J G_{5}, B J G_{6}, B J G_{7}, B J G_{8}, B J G_{9}, B J G_{10}$, and $B J G_{11}$, respectively.

\subsection{Progenitors and descendants}

We obtained the halo masses where our $B J G K$ samples reside (see Fig. 8). The galaxy samples of similar $K$-band absolute magnitude reside in halos of $10^{12.5 \pm 0.2} M_{\odot}$. In terms of progenitors and descendants for the median halo mass, the progenitors are the LBGs with $R<25$ at $z \sim 3$ (Adelberger et al. 2005; Hildebrandt et al. 2007), the $s B z K$ galaxies with $K \leq 23$ at $z \sim 2$ (Hayashi et al. 2007; Hartley et al. 2008; McCracken et al. 2010; Lin et al. 2012) and the descendants in the local Universe are galaxies with luminosities around $2 L_{*}$ (see Fig. 7). For halos around the upper limit $10^{12.7} M_{\odot}$, we found as progenitors at $z \sim 3$ the LBGs (Hildebrandt et al. 2007) with $R \leq 24$ and at $z \sim 2$ the sBzK galaxies with $K \leq 23$ (Hartley et al. 2008; Lin et al. 2012). The descendants are galaxies with luminosities 


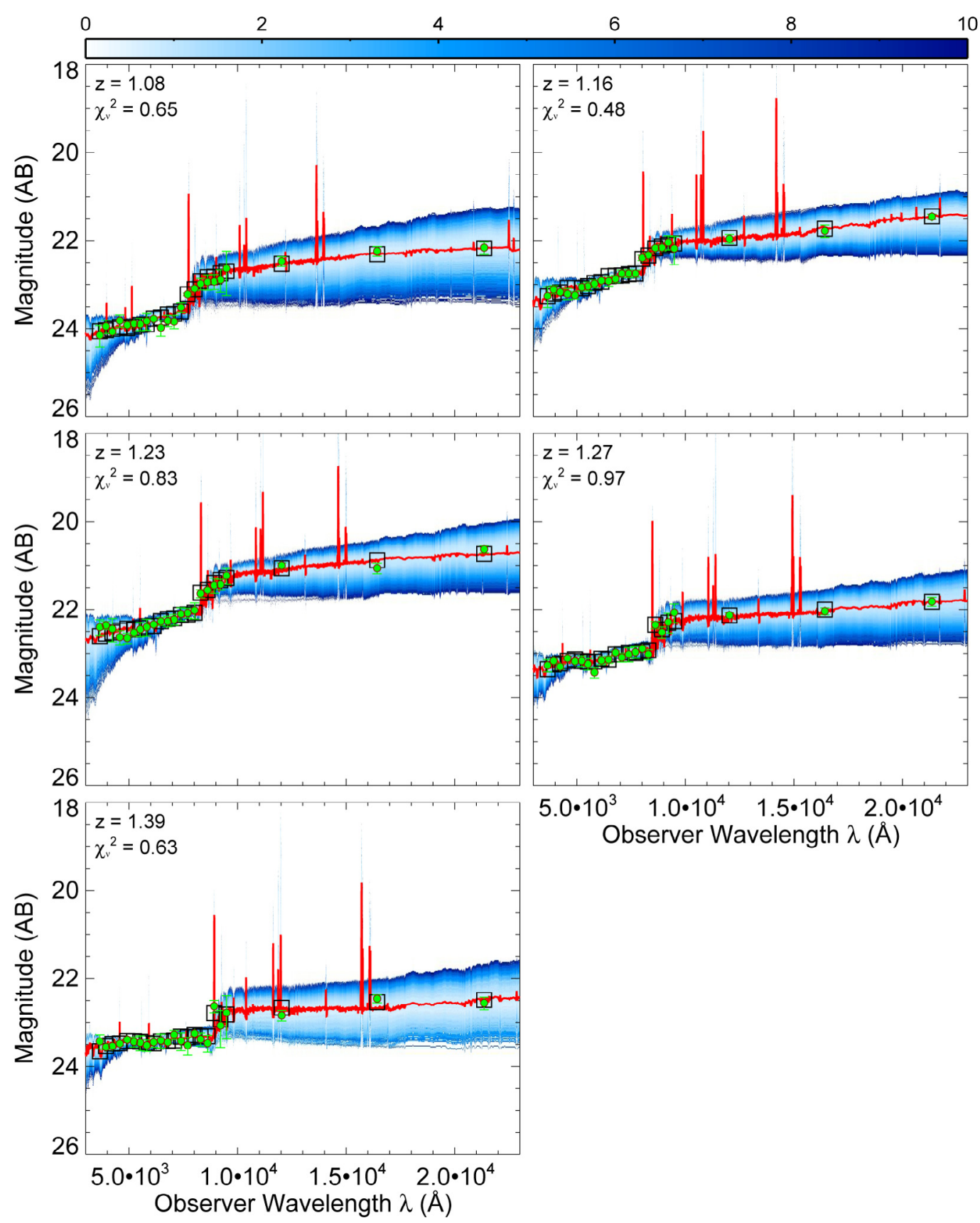

Fig. 10. Spectral energy distribution of a star-forming galaxy in the ALHAMBRA survey. From left to right and top to bottom, the panel shows the SED of a galaxy randomly picked from the $B J G_{7}, B J G_{8}, B J G_{9}, B J G_{10}$, and $B J G_{11}$ samples. The filled green dots show the ALHAMBRA photometric data. The red line shows the model that minimizes the $\chi^{2}$ found by iSEDfit, the minimum reduced $\chi^{2}$ is indicated in the upper left corner, in addition to the BPZ photometric redshift. The black squares mark the ALHAMBRA photometry of the best model. The blue shading shows the universe of models, generated by iSEDfit (see Sect. 4.2), scaled by their reduced $\chi^{2}$. The color bar indicates the reduced $\chi^{2}$ scale.

around $3 L_{*}$. For the halos around the lower limit $10^{12.3} M_{\odot}$, the progenitors are the LBGs at $z \sim 4$ (Kashikawa et al. 2006; Ouchi et al. 2005) with I or $z \leq 27$, the LAE galaxies at $z \sim 3$ (Gawiser et al. 2007), and the sBzK galaxies (Hayashi et al. 2007) with $K<23$ at $z \sim 2$. The descendants are galaxies with luminosities around $1 L_{*}$. The increment of the median halo mass of $\sim 0.4$ dex, between $z=1.0$ and $z=0.5$, follows the observed mass increment in numerical simulations (Millennium, Fakhouri et al. 2010). This suggests that we trace the evolution of halos with masses of around $\sim 10^{12.5}$ from $z=1.0$ to $z=0.5$, and hence the evolution of the physical properties, as is shown in Fig. 6. So far, we argued based on clustering measurements that our five sets of galaxies $B J G K_{n<6}$ represent a coeval and homogeneous population of star-forming galaxies.

The bias and hence halo mass of the $B J G K$ samples is higher on average than those found in Hurtado et al. (2016) for starforming galaxies. For all redshift ranges studied, our correlation lengths are $20 \%$ higher than in their work, suggesting that we selected more massive galaxies. This might be due to the different galaxy selection methods and to the absolute magnitude thresholds used to define complete samples. Nevertheless, the main difference is caused by the method and the scale that we chose to calculate the bias and hence the halo mass. Hurtado et al. (2016) calculated the bias for the scale range $1.0<r<10 \mathrm{Mpc}$, while 
in this work we evaluated the bias on the scale $8 h^{-1} \mathrm{Mpc}$ to compare our results with previous works that also studied the progenitors and descendants of star-forming galaxies. By taking this approach, we obtained halo masses 0.7 dex higher than in (Hurtado et al. 2016).

\subsection{Physical properties}

We performed an accurate estimation of the physical properties derived from SED fitting (e.g., absolute magnitude, stellar mass, and star formation rate) and characterized each sample as a whole with the median of each of these properties. Figure 6 shows the median of the probability distribution of stellar mass, absolute magnitude, and star formation rate for the $B J G K_{n<6}$ (red squares) samples as a function of redshift. In the stellar mass panel, the dashed line shows the evolution of the stellar mass for galaxies with present-day stellar masses of $\log \left(M_{*}\right) \approx 10.7$, such as our Milky Way galaxy. The data are taken from the analysis of the CANDELS survey by van Dokkum et al. (2013). The observed mass growth determined by van Dokkum et al. (2013), which slightly increase by a factor of $0.1 \mathrm{dex}$ from $z=1$ to $z=0.5$, agrees well with the flat behavior found in this work. In the star formation rate panel, the dashed line shows the evolution of the implied star formation rate that is caused by the evolution of the stellar mass of galaxies with present-day stellar masses of $\log \left(M_{*}\right) \approx 10.7$, such as our Milky Way, also taken from van Dokkum et al. (2013). Our results are slightly lower, suggesting that the SFR is sufficient to account for the increment in stellar mass between $z=1$ and $z=0.5$ and that major mergers play a minor role in this redshift range. The evolution of the SFR and $s$ SFR resembles the expected main-sequence behavior of star-forming galaxies (Rodighiero et al. 2011). The BJGK evolution from $z \sim 1$ to $z=0$ of the $B$-band absolute luminosity agrees with the evolution measured by Tasca et al. (2014). They found that the contribution of disks to the total $B$-band luminosity decreases by $30 \%$ from $z \sim 1$ to $z=0$, while for the $B J G s$ the median $B$-band luminosity decreases by a factor of 0.5 dex. The distribution of galaxy ages obtained by iSEDfit has a large dispersion, but even so, its increment between two epochs corresponds to the Universe age increment indicated by the redshift. According to the models of Lagos et al. (2014), galaxies of properties similar to the $B J G$ s host most of the neutral gas at $0.5<z<1.5$. Hence the $B J G$ samples contain tentative targets to sample the neutral gas with submillimeter surveys.

\section{Conclusions}

We have selected eleven samples of star-forming galaxies by using a two-color technique based on the Bruzual \& Charlot (2003) models convolved with the ALHAMBRA filters. Using clustering arguments, we confirmed that five out of the eleven sets of galaxies, that is, the $B J G K_{n<6}$, represent a coeval and homogeneous population of star-forming galaxies. The properties derived from SED fitting, such as stellar mass, star formation rate, age, and absolute luminosity of each $B J G K_{n<6}$ sample were characterized as a whole, allowing us to study their putative evolution as a function of redshift. The main results are summarized below.

We tailored a two-color selection technique, based on the Bruzual \& Charlot (2003) models and the Balmer jump, that selects star-forming galaxies in the redshift range $0.5<z<$ 1.5. We selected eleven samples composed of Balmer-jump Galaxies, dubbed $B J G$. The number of photometric-redshift outliers in these color-selected samples increases with redshift, ranging from $1 \%$ to $15 \%$.

We created a subsample of $B J G$, dubbed $B J G K_{n}$ with $n<6$, which considered only the $B J G_{n<6}$ galaxies brighter than $K_{\text {abs }} \sim$ -21.2 . The stellar mass of the $B J G K$ samples changes almost not at all with redshift,suggesting that major mergers played a minor role in the evolution of the $B J G K$ galaxies. The SFR evolution accounts for the small variations of the stellar mass, from $z \sim 1$ to $z \sim 0.5$, suggesting that star formation and minor mergers are the main channels of mass assembly. Although the distribution of galaxy ages obtained by iSEDfit has a large dispersion, the evolution of the galaxy age agrees with the evolution of the Universe age.

The $B J G K_{n<6}$ samples reside in halos of $10^{12.5 \pm 0.2} M_{\odot}$, whose progenitors are the LBGs with $R \leq 24$ at $z \sim 3$ (Hildebrandt et al. 2007 ) and the $s B z K$ galaxies (Lin et al. 2012; Hartley et al. 2008; McCracken et al. 2010) with $K \leq 23$ at $z \sim 2$. The descendants in the local Universe are galaxies with luminosities around 1-3 $L_{*}$ (for details see Fig. 7).

The similar increment of the median halo mass between $z=1.0$ and $z=0.5$ of our observational results and numerical simulations (Millennium, Fakhouri et al. 2010) suggests that we trace the evolution of halos of masses around $\sim 10^{12.5}$ from $z=1.0$ to $z=0.5$, and hence the putative evolution of the physical properties of the galaxies hosted by these halos (see Fig. 6).

The homogeneous coverage of the ALHAMBRA optical bands from $R_{9}$ to $z_{20}$ allows us to trace the evolution of the baryonic processes occurring on star-forming galaxies from $z \sim 1.0$ to $z \sim 0.5$, which reside in halos of masses $\sim 10^{12.5} h^{-1} M_{\odot}$. This roughly corresponds to a stellar mass upper (lower) limit of $\sim 10^{11} M_{\odot}\left(10^{10} M_{\odot}\right)$. Deeper ALHAMBRA data and nearinfrared selected catalogs would allow us to study the evolution of the physical properties in halos of masses lower than $10^{12.5} M_{\odot}$ from $z \sim 0.5$ to $z \sim 1.5$.

Acknowledgements. The work presented in this paper is based on observations taken at the Centro Astronómico Hispano Alemán (CAHA) at Calar Alto, operated jointly by the Max-Planck-Institut für Astronomie and the Instituto de Astrofísica de Andalucía (IAA-CSIC). P.T. and A.M.A. acknowledge support from FONDECYT 3140542 and FONDECYT 3160776, respectively. L.I., A.M.A., P.T., S.G., N.P. acknowledge support from Basal-CATA PFB-06/2007. E.J.A. acknowledges support from the Spanish Ministry for Economy and Competitiveness and FEDER funds through grant AYA201340611-P. A.F.S., V.J.M. and P.A.M. acknowledge partial financial support from the Spanish Ministry for Economy and Competitiveness and FEDER funds through grant AYA2013-48623-C2-2, and from Generalitat Valenciana through project PrometeoII 2014/060. B.A. acknowledges received funding from the European Union's Horizon 2020 research and innovation programme under the Marie Sklodowska-Curie grant agreement No. 656354.

\section{References}

Arnalte-Mur, P., Martínez, V. J., Norberg, P., et al. 2014, MNRAS, 441, 1783 Adelberger, K., Steidel, C. C., Pettini, M., et al. 2005, ApJ, 619, 697

Aparicio Villegas, T., Alfaro, E. J., Cabrera-Caño, J., et al. 2010, AJ, 139, 1242

Benitez, N., Moles, M., Aguerri, J. A. L., et al. 2009, ApJ, 692, L5

Blanc, G., Lira, P., Barrientos, L. F., et al. 2008, ApJ, 681, 1099

Bruzual, G., \& Charlot, S. 2003, MNRAS, 344, 1000

Bouwens, R. 2014, ApJ, 795, 126

Bouwens, R. 2015, ApJ, 803, 34

Cabré, A., Fosalba, P., Gaztañaga, E., \& Manera, M. 2007, MNRAS, 381, 1347

Chabrier, G. 2003, PASP, 115, 763

Campbell, D. J. R., Baugh, C. M., Mitchell, P. D., et al. 2015, MNRAS, 452, 852

Charlot, S., \& Fall, S. M. 2000, ApJ, 539, 718

Conroy, C., \& Gunn, J. E. 2010, ApJ, 712, 833

Conroy, C., Gunn, J. E., \& White, M. 2009, ApJ, 699, 486

Contreras, S., Baugh, C. M., Norberg, P., \& Padilla, N. 2013, MNRAS, 432, 2717 
Cristóbal-Hornillos, D., Aguerri, J. A. L., Moles, M., et al. 2009, ApJ, 696, 1554 Daddi, E., Cimatti, A., Renzini, A., et al. 2004, ApJ, 617, 746

Daddi, E., Elbaz, D., Walter, F., et al. 2010, ApJ, 714, L118

Davé, R., Finlator, K., Oppenheimer, B. D., et al. 2010, MNRAS, 416, 1354

Dey, A., Soifer, B. T., Desai, V., et al. 2008, AJ, 677, 943

Elbaz, D., Dickinson, M., Hwang, H. S., et al. 2011, A\&A, 533, A119

Fakhouri, O., Ma, C., \& Boylan-Kolchin, M. 2010, MNRAS, 406, 2267

Finoguenov, A., Guzzo, L., Hasinger, G., et al. 2007, ApJS, 172, 182

Gawiser, Francke, H., Lai, K., et al. 2007, ApJ, 671, 278

Guaita, L., Gawiser, E., Padilla, N., et al. 2010, ApJ, 714, 255

Guzzo, L., Cassata, P., Finoguenov, A., et al. 2007, ApJS, 172, 254

Hayashi, M., Shimasaku, K., Motohara, K., et al. 2007, AJ, 660, 72

Hartley, W. G., Lane, K. P., Almaini, O., et al. 2008, MNRAS, 391, 1301

Hildebrandt, H., Pielorz, J., Erben, T., et al. 2007, A\&A, 462, 865

Hurtado-Gil, L. L., Arnalte-Mur, P., Martínez, V. J., et al. 2016, ApJ, 818, 174

Infante, L. 1994, A\&A, 282, 353

Infante, L., Zheng, W., Laporte, N., et al. 2015, ApJ, 815, 18

Kashikawa, N., Yoshida, M., Shimasaku, K., et al. 2006, AJ, 637, 631

Kovac, K., Somerville, R. S., Rhoads, J. E., Malhotra, S., \& Wang, J. 2007, ApJ, 668,15

Lagos, C. P., Baugh, C. M., Zwaan, M. A., et al. 2014, MNRAS, 440, 920

Lejeune, T., Cuisinier, F., \& Buser, R. 1997, A\&AS, 125, 229

Lejeune, T., Cuisinier, F., \& Buser, R. 1998, A\&AS, 130, 65

Lilly, S., Carollo, C. M., Pipino, A., Renzini, A., \& Peng, Y. 2013, ApJ, 772, 119

Lin, L., et al. 2012, AJ, 756

Madau, P., \& Dickinson, M. 2014, ARA\&A, 52, 415

Mannucci, F., Cresci, G., Maiolino, R., Marconi, A., \& Gnerucci, A. 2010, MNRAS, 408, 2115

McCracken, H. J., Capak, P., Salvato, M., et al. 2010, AJ, 708, 202

Moles, M., Benítez, N., Aguerri, J. A. L., et al. 2008, AJ, 136, 1325

Molino, A., Benitez, N., Moles, M., et al. 2013, MNRAS, 441, 2891

Moustakas, J., Coil, A. L., Aird, J., et al. 2013, AJ, 767, 50
Norberg, P. Baugh, C. M., Gaztañaga, E., \& Croton, D. J. 2009, MNRAS, 396, 19

Norberg, P. Gaztañaga, E., Baugh, C. M., \& Croton, D. J. 2011, MNRAS, 418, 2435

Ouchi, M., Hamana, T., Shimasaku, K., et al. 2005, AJ, 635, L117

Padilla, N., Christlein, D., Gawiser, E., Marchesini, D., et al. 2011, A\&A, 531, A142

Padilla, N., Salazar-Albornoz, S., Contreras, S., Cora, S. A., \& Ruiz, A. N. 2014 MNRAS, 443, 2801

Peebles, P. J. E. 1980, The large scale structure of the Universe (Princeton: Princeton University Press), 435

Quadri, R., van Dokkum, P., Gawiser, E., et al. 2007, AJ, 654, 138

Rodighiero, G., Daddi, E., Baronchelli, I., et al. 2011, AJ, 739, L40

Sanchez, S., Rosales-Ortega, F. F., Iglesias-Páramo, J.,et al. 2014, A\&A, 563, A49

Sanchez-Blázquez, P., Peletier, R. F., Jiménez-Vicente, J., et al. 2006, MNRAS, 371,703

Scoville, N., Aussel, H., Benson, A., et al. 2007, ApJS, 172, 150

Sheth, R. K., Mo, H. J., \& Tormen, G. 2001, MNRAS, 323, 1

Steidel, C., Giavalisco, M., Pettini, M., Dickinson, M., \& Adelberger, K. L. 1996, ApJ, 462, L17

Steidel, C., Shapley, A. E., Pettini, M., et al. 2004, ApJ, 604, 534

Tasca, L. A. M., Tresse, L., Le Fèvre, O., et al. 2014, A\&A, 564, L12

Tomczak, A., Quadri, R. F., Tran, K.-V. H., et al. 2014, AJ, 783, 85

Troncoso, P., Maiolino, R., Sommariva, V., et al. 2014, A\&A, 563, A58

van Dokkum, P., Leja, J., Nelson, E. J., et al. 2013, AJ, 771, L35

Viironen, K., Marín-Franch, A., López-Sanjuan, C., et al. 2015, A\&A, 576, A25

Westera, P., Lejeune, T., Buser, R., Cuisinier, F., \& Bruzual, G. 2002, A\&A, 381, 524

Yoshida, M., Shimasaku, K., Ouchi, M., et al. 2008, AJ, 679, 269

Zehavi, I., Blanton, M. R., Frieman, J. A., et al. 2002, ApJ, 571, 172

Zehavi, I., Zheng, Z., Weinberg, D. H., et al. 2011, AJ, 736, 59 